\title{
Intermédialités
}

Histoire et théorie des arts, des lettres et des techniques

Intermediality

History and Theory of the Arts, Literature and Technologies

\section{Temporaliser la photographie : Marker, Varda, Wenders-Salgado}

\section{Antoine Constantin Caille}

Numéro 33, printemps 2019

restituer (le temps)

rendering (time)

URI : https://id.erudit.org/iderudit/1065013ar

DOI : https://doi.org/10.7202/1065013ar

Aller au sommaire du numéro

Éditeur(s)

Revue intermédialités

ISSN

1920-3136 (numérique)

Découvrir la revue

Citer cet article

Caille, A. C. (2019). Temporaliser la photographie : Marker, Varda,

Wenders-Salgado. Intermédialités / Intermediality, (33).

https://doi.org/10.7202/1065013ar
Résumé de l'article

À travers trois films qui reposent sur des photographies - Si j'avais quatre dromadaires de Chris Marker (1966), Ulysse d'Agnès Varda (1982) et Le sel de la Terre de Wim Wenders et Juliano Ribeiro Salgado (2014) - , cette contribution interroge la valeur et le sens de la restitution du temps. Nous considérons la part que joue dans le film la restitution du contexte bio-historiographique de la photographie, mais aussi la capacité de l'oeuvre photocinématographique à rendre sensible le temps. De quel temps s'agit-il alors ? Celui du réel photographié ou celui inventé par l'oeuvre ? Peut-on restituer ce temps réel et singulier de l'instant photographié, et est-ce cette restitution qui donnera tout son sens à la photographie ? Quelle importance faut-il donner à la singularité du moment ? L'enjeu de la restitution du temps ne dépasse-t-il pas celui de la restitution de cette singularité ? 


\title{
Temporaliser la photographie : Marker, Varda, Wenders-Salgado
}

\author{
Antoine Constantin Callle
}

uand un film montre une photographie - différenciée des photogrammes qui le constituent -, il ne fait pas que la reproduire; il opère sur elle, à plusieurs égards, une temporalisation. Premièrement, la durée pendant laquelle la photographie est exposée au regard du spectateur — ou inversement, le regard du spectateur à la photographie - est déterminée par le réalisateur du film (ou son monteur), et non par le spectateur lui-même, comme c'est le cas lorsqu'on feuillette un album de photos ou lorsqu'on visite une salle d'exposition. Deuxièmement, la photographie est insérée dans la continuité temporelle du film. Si un album, un livre, un site de photographies ou une exposition ordonnancent leur espace et donc prédéterminent l'ordre de réception sans forcément contraindre le spectateur, le film montre la photographie dans une durée, la sienne. Troisièmement, renforçant ces deux premiers aspects, la voix d'un narrateur-commentateur peut jouer au moins deux fonctions dans cette temporalisation : expliciter la place de la photographie dans le film et ses liens avec les autres éléments ou moments du film; restituer le contexte biographique et/ou sociohistorique dans lequel la photographie a été prise.

La restitution (du temps) n'est qu'un des aspects de la temporalisation. De même, une conception limitant la restitution du temps à une description du contexte, qu'il soit celui du film ou d'un ordre plus général, est certainement restrictive. Elle est en effet oublieuse de l'aspect qualitatif du temps, et du travail artistique qui entreprend de le rendre perceptible. En se référant à la définition d'usage, restituer ne serait pas seulement resituer, mais « redonner ce qui a été perdu ${ }^{\mathrm{I}} »$. Toutefois, nous

${ }^{1}$ http://www.cnrtl.fr/definition/restituer (consultation le 30 juin 20I9). 
envisageons cette modalité de la restitution comme étant aussi distincte du travail artistique consistant à produire une nouvelle temporalité, propre à l'expérience $d u$ visionnement de l'œuvre photocinématographique. Il s'agit d'étudier le rapport, voire l'entremêlement, entre une restitution informative et une restitution artistique, pouvant impliquer d'autres aspects de temporalisation, et ce, dans la perspective de donner à expérimenter un moment passé.

Afin de mieux discerner les enjeux du problème qui nous retient, nous examinerons trois films : Si j'avais quatre dromadaires (1966) de Chris Marker, Ulysse (1982) d'Agnès Varda, et Le sel de la Terre (2014) de Wim Wenders et Juliano Ribeiro Salgado ${ }^{2}$. Notre analyse tentera de restituer la temporalité propre à chacune de ces œuvres, en portant attention à la façon dont une temporalité artistiquement construite travaille elle-même à restituer celle(s) de l'histoire/Histoire des photographies. Ce faisant, nous découvrirons que chacune de ces œuvres élabore, en contrepoint de la restitution du temps historique, une pensée de l'atemporel ou de l'anhistorique, qui, en retour, mène à interroger le sens de la restitution que nous visons à expliciter. Nous emprunterons certains outils conceptuels d'analyse filmique à Michel Chion et de narratologie à Mikhail Bakhtine, Roland Barthes et Gérard Genette, nous inspirant en outre de distinctions opérées par Edmund Husserl et Bernard Stiegler entre trois types de rétention mémorielle, rejoignant ainsi des recherches contemporaines sur la combinaison médiatique photocinématographique.

\section{PRÉSENTATION DU CORPUS}

Si j'avais quatre dromadaires se distingue principalement des deux autres films par le fait que, sur le plan de l'image, il est entièrement composé de photographies. Les commentateurs restent ainsi acousmatiques, c'est-à-dire qu'ils demeurent invisibles. La restitution du contexte historique et biographique tient particulièrement à l'une des trois voix, celle qui s'identifie comme appartenant au photographe joué par Pierre Vaneck, bien que Marker soit l'auteur réel des photographies. Étudier le travail qu'opère Marker sur le temps en reliant des espaces géographiques disjoints et distants dans une narration hétérologique permettra de faire ressortir l'évolution philosophique et la cohérence d'un propos mimant la conversation improvisée.

${ }^{2}$ Chris Marker, Si j'avais quatre dromadaires, Les Mutins de Pangée, 1966; Agnès Varda, Varda tous courts, Short Films Collection, 1982; Wim Wenders et Juliano Ribeiro Salgado, Le sel de la Terre, France Télévision Distribution, 2014. 
Dans Ulysse, le choix narratif de Varda est sensiblement différent: la réalisatrice se met en scène, à la recherche du sens d'une seule de ses photographies. Elle se montre très peu, mais c'est sa propre voix qui prédomine, à l'occasion de ses retrouvailles avec les modèles de la photographie qu'elle filme presque trois décennies plus tard. Son enquête explore ainsi différentes pistes herméneutiques, de la plus naïve, qui consiste à réveiller les souvenirs pour restituer le temps et ainsi (re)trouver le sens de l'œuvre, à la plus ambitieuse, à travers une injonction à percevoir la scène comme un mythe (Ulysse...), un fragment d'éternité, un symbole du temps (les trois âges).

Le sel de la Terre offre un dispositif narratif plus commun : un cinéaste (Wim Wenders) et un vidéaste (Juliano Ribeiro Salgado) vont à la rencontre d'un photographe (Sebastião Salgado), chacun ayant son rôle, clairement circonscrit. Le film est à la fois une biographie de Sebastião Salgado et à travers elle une exploration historiographique des grandes crises de la seconde moitié du vingtième siècle. De même que dans les deux films précédents, la restitution du contexte sociohistorique n'est pas qu'une ré-exploration du passé, elle a aussi pour fonction de permettre une appréciation du travail de l'artiste, notamment dans sa tentative de restituer dans sa spécificité qualitative le temps propre à un style de vie ou à une situation. Pourtant, là encore, le commentaire du photographe enjoint au spectateur de percevoir une dimension mythique, quasi extratemporelle : l'humain contre les éléments, l'humain et la survie, l'humain face au cataclysme qu'il a lui-même causé... Nous verrons comment ce film valorise tour à tour la perception de la singularité et la compréhension du général, et aboutit à une célébration d'une certaine idée de l'éternité synonyme d'une sortie de l'historicité.

\section{SI J'AVAIS QUATRE DROMADAIRES: DE LA CRÉATION DE TEMPORALITÉS À L'ATTENTION AU MOUVEMENT DU MONDE}

Dans son article « Les usages de la photographie : Il est six heures sur toute la Terre », Filippa Ramos résume bien le concept de l'œuvre en disant que Marker « transpose au cinéma l'expérience de regarder à plusieurs un album de voyage ». Elle y révèle que le film est apparenté à un projet abandonné de livre-album et cite une description retrouvée dans une lettre de Marker : « Plutôt que des photos de voyages, elles [les photos] souhaitent être prises elles-mêmes pour un voyage : un voyage dans 
la diversité humaine 3 . » Ce voyage, finalement photocinématographique, commence par une réflexion sur la photographie comme pratique comparable à la chasse, dans une formule souvent reprise par la suite : « on traque, on vise, on tire, et puis clac, au lieu d'un mort, on fait un éternel $\gg($ I:06 - I:09).

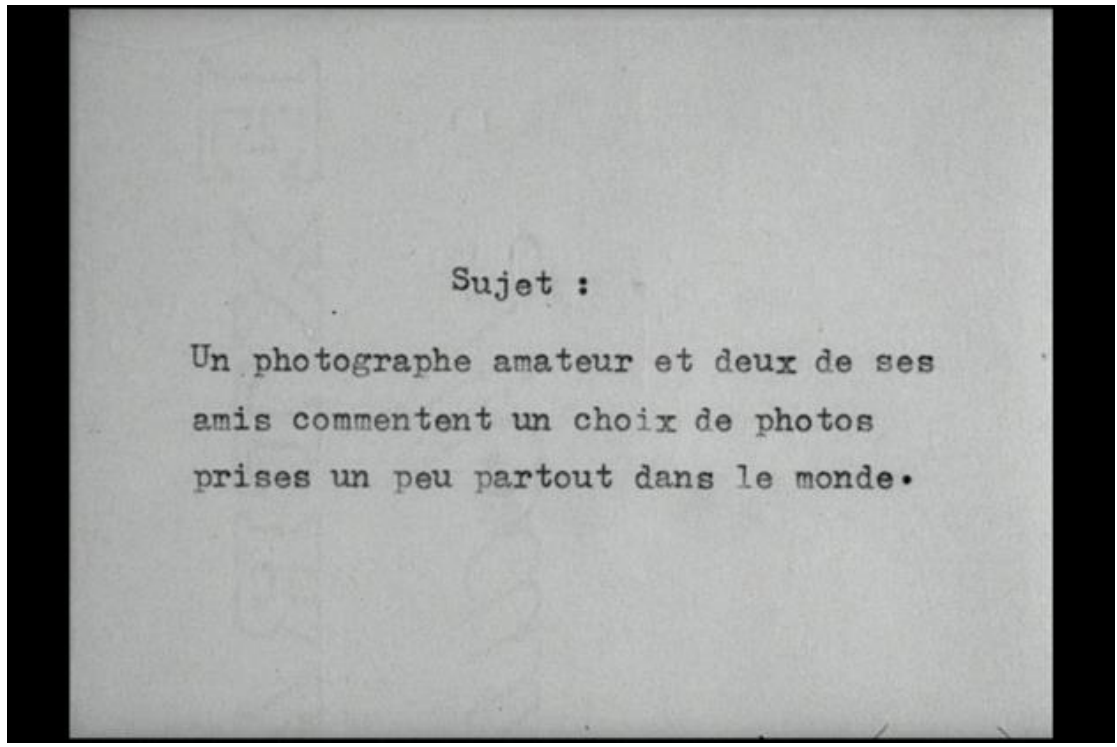

Fig. I : Photogramme du film Si j’avais quatre dromadaires, Chris Marker, 1966.

Très vite, il est donc question d'éternité, ou plus exactement de la photographie comme technique qui procure de l'éternité. Si ce thème initial n'est guère original, puisque l'expression figée «immortaliser quelqu'un » est bien connue, l'idée devient sensible grâce à la combinaison des deux médias : donner à voir une image fixe à travers un médium où l'on s'attend à une image en mouvement accentue l'impression d'éternisation de l'instant ${ }^{4}$. L'idée d'éternisation est reprise

3 Filipa Ramos, « Les usages de la photographie : Il est six heures sur toute la Terre », dans Christine Van Assche, Raymond Bellour et Jean-Michel Frodon (dir.), Chris Marker, Paris, Cinémathèque française, 20I8, p. 159-160.

4 François Niney a déjà traité des effets temporels de cette pratique intermédiale, notamment d'éternisation, de synchronie et d'anachronie. Voir François Niney, «Photo animée et photogramme arrêté : le "cinémarker", entre reprise et suspens du temps », dans Vincent Jacques (dir.), Chris.Marker.Photographie, Paris, Creaphis Éditions, coll. « Poche », 2018, p. I07-I23. Ramos aborde également cette question en offrant une bonne introduction à l'interartialité photocinématographique chez Marker et à ses enjeux temporels. 
ensuite à propos non plus des sujets représentés, mais du regard de l'artiste, puis de la photographie elle-même, donnant à penser un second dédoublement et un troisième.

- Et voilà encore quelque chose: un sculpteur a éternisé un certain visage avec un certain regard [lent zoom avant sur les yeux de la sculpture], et toi à ton tour, avec la photo, tu éternises ton propre regard sur ce regard.

- [Autre voix d'homme :] Et moi je regarde la photo qui regarde.
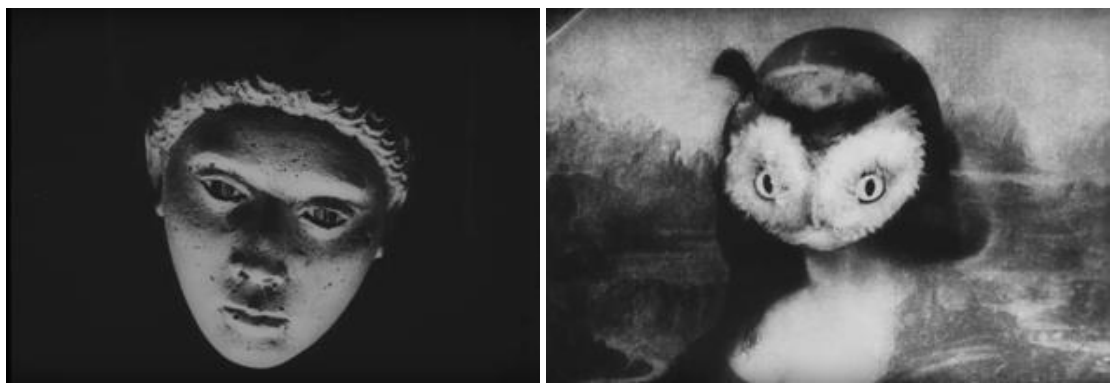

Fig. 2 et 3 : Photogrammes du film Si j'avais quatre dromadaires, Chris Marker, 1966.

Et nous, spectateurs, regardons ce film où un moi (une voix) regarde une photo qui regarde... Un nouveau niveau d'interartialités est atteint avec les photographies (filmées) d'œuvres d'art, en particulier de peintures et sculptures (voir les figures 2, 3 et 4), renforçant à la fois l'impression d'infinitisation de la perception et d'éternisation (puisqu'il s'agit maintenant de la fixation d'un objet qui est luimême essentiellement figé). Il s'ensuit logiquement des commentaires élaborant une critique de l'illusion réaliste, différenciant la photographie du réel qu'elle représente, la vie: «Il y a la vie, il y a son double, et la photo appartient au monde du double. » (I:56 - 2:0I) Cette thèse ontologique sur la photographie va dans le sens d'une mise en garde destinée aux spectateurs (le commentateur s'incluant) :

s Sur ce terme et sa différence avec celui d'intermédialité, voir notamment Rémy Besson, « Prolégomènes pour une définition de l'intermédialité », Cinemadoc, Imagines animées, archives visuelles et intermédialités, 2014, https://cinemadoc.hypotheses.org/2855 (consultation le 30 juin 2019). Rémy Besson y fait référence à l'article de Walter Moser, «L'interartialité : pour une archéologie de l'intermédialité » paru dans Marion Froger et Jürgen E. Müller (dir.), Intermédialité et socialité. Histoire et géographie d'un concept, Münster, Nodus Publikationen, 2007 p. 70. 
TEMPORALISER LA PHOTOGRAPHIE :

MARKER, VARDA, WENDERS-SALGADO

- D'ailleurs, c'est là qu'il y a un piège. À force de t'approcher des visages lla caméra opère un zoom avant sur un portrait byperréaliste à partir d'un cadrage qui laisse voir le «réel» qui l'entoure], tu as l'impression que tu participes à leur vie et à leur mort de visages vivants, de visages humains. C'est pas vrai. Si tu participes à quelque chose, c'est à leur vie et à leur mort d'images. $(2: 02-2: 20)$
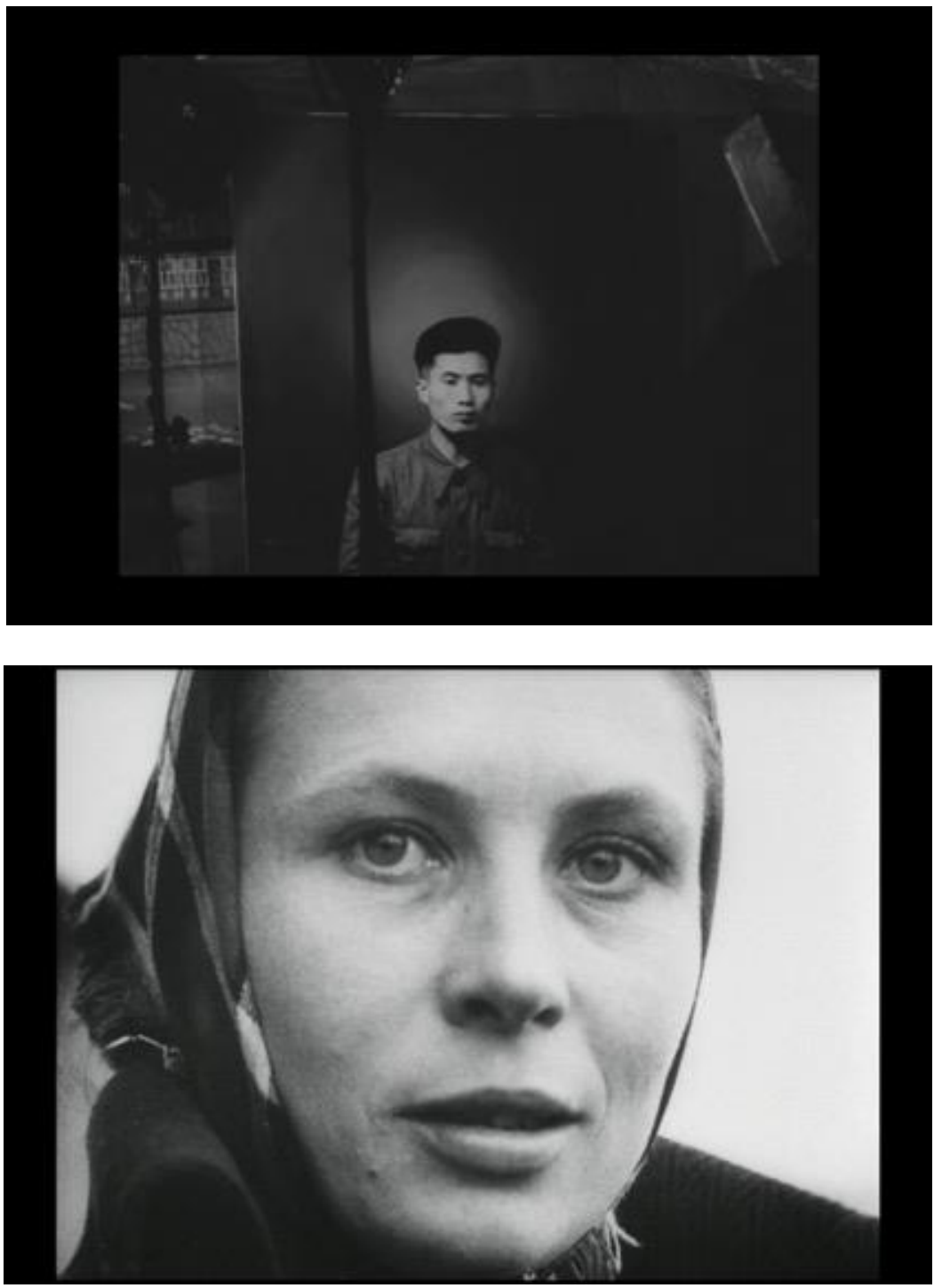

Fig. 4 et 5 : Photogrammes du film Si j'avais quatre dromadaires, Chris Marker, 1966. 
Cette mise en garde à la Magritte nous invite à prévenir notre tendance à « passer à travers » le médium : bien qu'elle soit au moins double ici (photographie et cinéma), la médialité disparaît par la force d'expression d'un visage pris « sur le vif » (voir la figure 5). Ici la question de l'émotion suscitée par l'image de la personne photographiée se pose de façon inverse à celle suscitée par le personnage de fiction littéraire $^{6}$, non seulement parce que la personne a bel et bien existé, mais aussi parce que nous n'en saisissons qu'un instant, la soustrayant à sa temporalitét. Par l'ajout d'une narration donnant à connaître des détails sur la vie de cette personne, comme c'est le cas quelquefois dans ce film et plus encore dans les deux autres, et par l'ajout de séquences filmées incluant cette personne - comme dans Ulysse et très partiellement dans Le sel de la Terre - le cinéaste peut changer la donne : suppléer au manque de temporalité narrative de la photographie. Mais à ce moment du film, Marker choisit de limiter l'expérience du spectateur à celle de l'instantanéité, paradoxalement éprouvée dans la durée qu'il a décidée au montage. Qui plus est, parler de « la vie et de la mort des visages en tant qu'images » apparaît comme une mise en garde relevant d'une logique qui consiste à dissocier l'œuvre de ce qu'elle représente et à faire ressortir l'étrange atemporalité des photographies.

La dissociation fait le jeu du commentaire: le jeu introduit entre les photographies et les événements capturés permet de s'amuser à créer une simultanéité impossible autrement qu'artistiquement. Le commentateur personnifiant le photographe fait état de sa fascination pour la simultanéité magique (et factice) qu'opère un certain type de narration sur des images hétéroclites. Tout en dénonçant cette magie, le film la renforce, la rend plus sensible et explicite — notamment à l'aide de fondus enchaînés poétiques, surréalistes par surimpression (voir les figures 6 et 7 ).

\footnotetext{
6 Par comparaison, voir les remarques d'Alexandre Gefen dans son article « L'émotion dans la fiction » dans Françoise Lavocat, Catherine Courtet, Mireille Besson, Alain Viala (dir.), Corps en scènes, Paris, CNRS éditions, 2015, disponible en ligne: https://hal.archivesouvertes.fr/hal-oi624109/document (consultation le 26 septembre 2019).

7 Sur ce point, voir les remarques de Niney, 2018.
} 

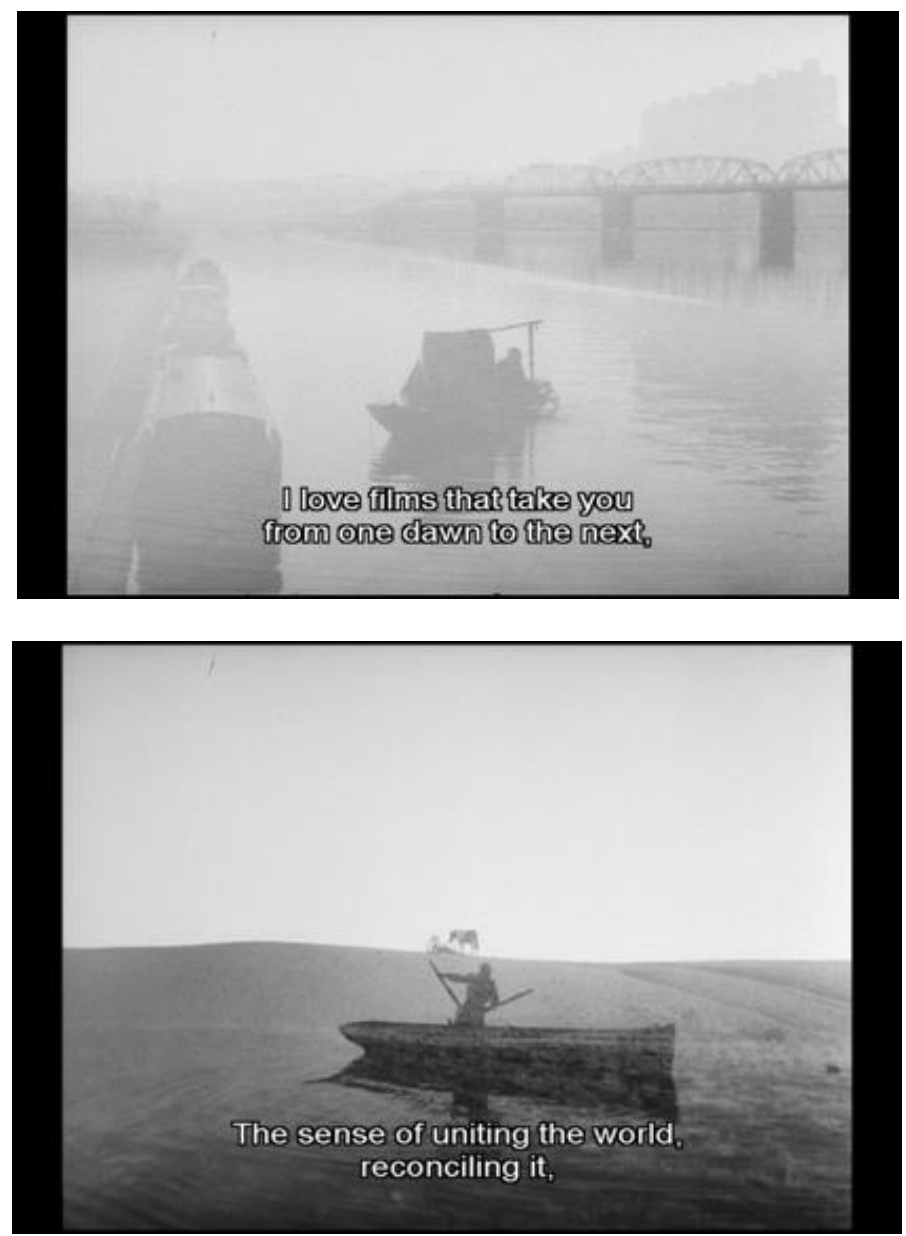

Fig. 6 et 7 : Photogrammes du film Si j'avais quatre dromadaires, Chris Marker, 1966.

La narration tire parti d'une ambiguïté quant à ce qui est simultané, simulant une simultanéité des événements là où nous savons qu'il ne peut s'agir que d'un effet du montage. En plus de l'impression d'ubiquité produite par la surimpression, de multiples jeux langagiers établissent des rimes et des chiasmes entre des endroits distants. Il y a notamment une suite de raccords faits sur la base de consonances entre les noms de lieux, sur laquelle se greffe un jeu de ressemblances et dissemblances entre les paysages et les situations. Il ne s'agit non pas d'une restitution en vrac mais d'une réorganisation libérée de la temporalité première, multipliant les effets de sens. On peut donc distinguer différents niveaux de perception : celui de la prise de vue impliquant succession et déplacement physiques, celui du montage amenant l'idée de 
réorganisation mentale, et rendant possibles la simultanéité, l'ubiquité, et même une forme de hors-temps, celui de la représentation du monde du point de vue de son unicité.

Cette simultanéité artificielle, qui ne s'offre pas comme une description rigoureuse du réel ${ }^{8}$, donne à contempler la diversité des événements terrestres, leur dissonante cohabitation, leur lieu à la fois commun et distant — «le » monde. L'imagination du photographe-réalisateur-commentateur (écrivain du dialogue), qui relie/relit les images par ces jeux de rimes, de ressemblances et de dissonances, témoigne à sa manière de la richesse d'une telle contemporanéité. Cette fictive restitution du temps à partir d'événements réels fait du dispositif photographiecinéma un art de la création de temporalités susceptible, sinon d'approcher la complexité du réel, du moins d'en complexifier notre compréhension. Elle réalise ce que le narrateur de Sans Soleil (Marker, 1983) déclare avoir été «l'enjeu du XX siècle $»:$ : la cohabitation des temps ${ }^{9} »$.

Le procédé consistant à produire une impression de simultanéité n'est qu'un des nombreux procédés narratifs qui foisonnent au cours de ce moyen métrage expérimental. Une sorte de tour de passe-passe est opéré à l'aide des temps grammaticaux : l'indicatif présent produit cette impression de simultanéité («il est six heures », « le jour se lève »), puis d'autres temps font irruption, multipliant les modalités de narration. Le subterfuge le plus remarquable consiste à renverser la modalité du commentaire : les commentateurs se mettent à suivre leurs propres pensées ou ressouvenirs, à philosopher librement, à divaguer, et ce n'est alors plus leurs propos qui commentent les photographies, mais les photographies qui coréalisent leurs pensées. La préséance et la prévenance de l'image sur le discours sont déstabilisées par des propos qui ne cessent de prendre le large par rapport à la fonction de restitution du commentaire ${ }^{\mathrm{IO}}$. Le travail de restitution se limite souvent à ce qu'il est nécessaire de donner à connaître pour développer une pensée-images, une penséeimages-mouvement à partir d'instantanés.

L'élaboration de pensées au moment fictif du visionnement des photos par les commentateurs paraît prévaloir sur la restitution informative ou qualitative $d u$

\footnotetext{
${ }^{8}$ L'idée d'une simultanéité entre événements se produisant en des lieux différents est périmée au moins depuis l'article sur la relativité générale d'Albert Einstein. La théorie de la relativité restreinte et générale, Malakoff, Dunod, 2012 [1905].

9 Chris Marker, Sans Soleil, Argos Films, 1983.

1o « Le texte ne commente pas plus les images que les images n'illustrent le texte », écrivait Chris Marker dans Le Dépays, Paris, Éditions Herscher, 1982, p. I.
} 
moment qu'elles ont fixé. Cette impression est renforcée par l'aspect hétérophonique (la multiplicité des voix ${ }^{\mathrm{II}}$ ) du commentaire. La pensée se développe dans un dialogue à trois voix, se relayant, s'interpellant, se répondant, se différenciant, en un subtil « tressage $\mathrm{I}^{\mathrm{I} 2}$ ». Des monologues imposants ou divagateurs accaparent une séquence, forment et occupent leurs propres espaces-temps, baignent une série de photographies de leur subjectivité. Que la spécificité du style de Marker soit de faire naître des «messages personnels ${ }^{13}$ » et des considérations philosophiques plus ou moins rêveuses ou poétiques d'un travail d'archiviste du réel historique ${ }^{\mathrm{I} 4}$ ne requiert pas une restitution informative continue. La transversalité entre les lieux qui était le propre du segment simulant la simultanéité est abandonnée au profit d'une organisation des photographies par voyages ou zones culturelles. Cela permet de partager le commentaire informatif entre plusieurs photographies. La restitution qualitative du temps se fait le plus souvent tout au long d'une séquence, comme dans celle du couvent russe en Grèce (19:45 - 23:30), qui donne à sentir l'atmosphère de temps volontairement arrêté particulière à ce lieu de culte.

Si les commentaires ont quelquefois tendance à affirmer des « vérités » quasi atemporelles sur certaines cultures (l'âme russe, la liberté cubaine, la beauté coréenne...), en contrepartie les effets d'atemporalité obtenus grâce à l'art photographique sont désormais soigneusement démentis par des commentaires rétablissant l'historicité du moment photographié (voir les figures 8 et 9) :

"Voir Mikhail Bakhtine, La poétique de Dostö̈evski, Paris, Seuil, 197o [1929].

${ }^{12}$ Ce concept est défini par Michel Chion comme suit : «Il y a tressage des éléments sonores entre eux, ou d'un élément sonore de nature verbale, musicale, etc., avec tout ou partie de l'image, quand il y a réponse entre l'un et l'autre, transfert, impression que l'un est continué et relayé (même sous forme de démenti) par l'autre - ce qui se produit notamment dans certaines formes particulières du rapport dit/montré (scansion, contraste, voire contradiction). » Chion désigne cinq modalités du rapport dit/montré, soit le tressage, la scansion, le contraste, la contracition et le contrepoint. Voir « Glossaire », 2006, disponible sur le site de la revue Langue-Tempête, http://www.lampe-tempete.fr/ChionGlossaire.html (consultation le zo juin 2019).

13 Voir l'essai poético-biographique de Maroussia Vossen, rempli d'anecdotes qui permettent de mieux connaître la personnalité et le travail de son père adoptif, Chris Marker (le livre impossible), Paris, Le Tripode, 2016, p. 65.

${ }^{14}$ Sur l'importance de l'archive chez Marker, voir en particulier l'article de Raymond Bellour «L'archiviste » dans le collectif Chris Marker, 2018, p. I04-IO9. L'article donne une idée de l'extraordinaire travail, obsessionnel et parfois déroutant, de collecte d'informations et d'images opéré par Marker tout au long de sa carrière. 
- Les Coréens, c'était aussi la beauté; c'était aussi la science - une science qui va très loin, qui remonte au temps où ils avaient inventé l'imprimerie, avant Gutenberg, le cuirassé, avant Potemkine, les mille manières de vivre ou de peindre que les Japonais leur ont pris, avec la reconnaissance que l'on sait. Je ne joue pas ici la Corée éternelle contre la Corée populaire. Ces danses intemporelles, je les ai saisies à l'heure de la pause, dans une usine, dans un club d'ouvriers au bord du Pacifique - dans le temps. (31:55-32:35)
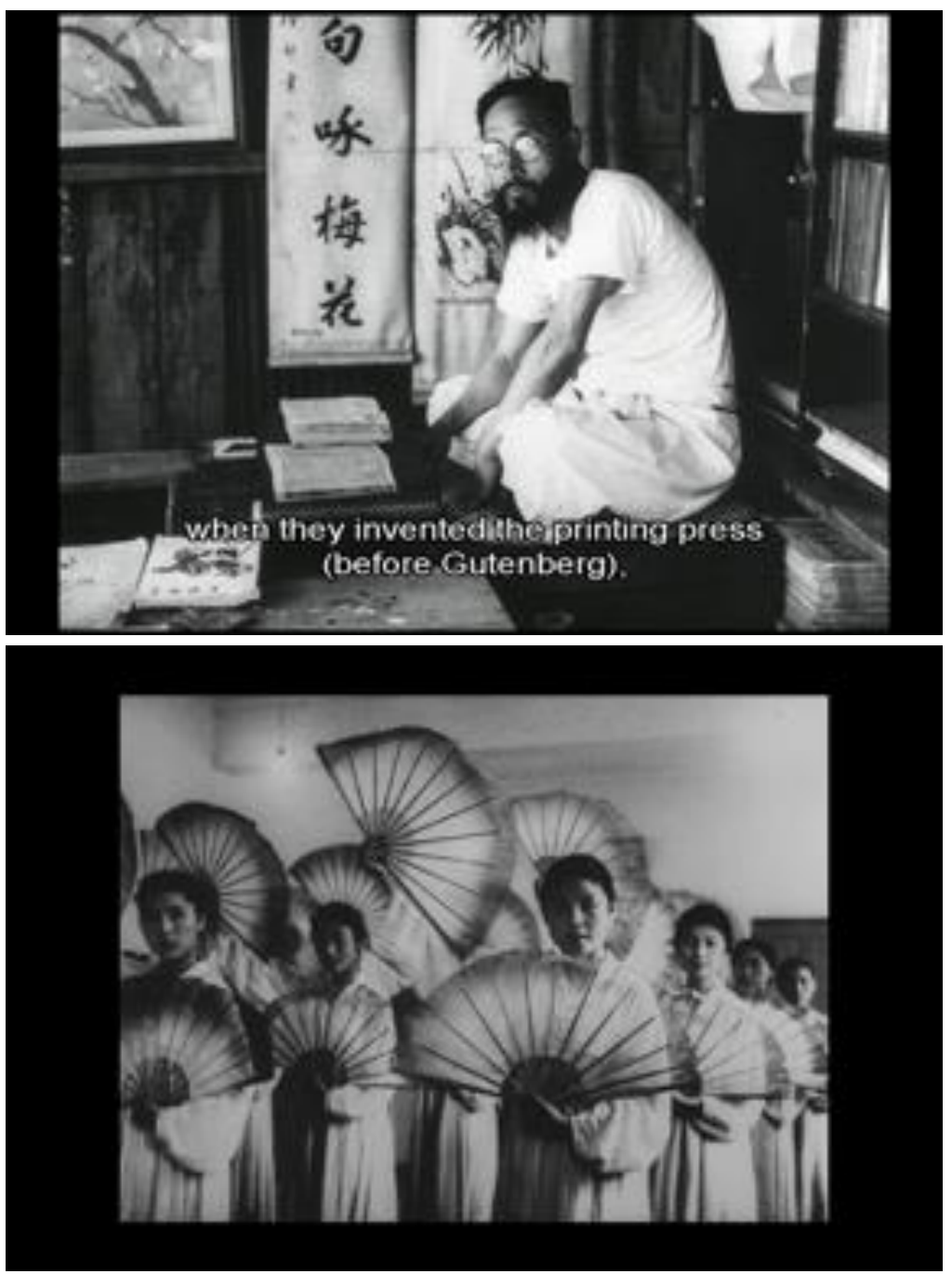

Fig. 8 et 9 : Photogrammes du film Si j'avais quatre dromadaires, Chris Marker, 1966. 
À mesure que le dialogue avance, un point de vue sur l'histoire se constitue dans l'interlocution. L'hétérophonie ne donne pas lieu ici à un perspectivisme ou à une véritable hétérologie ( « un discours de l'autre, sur l'autre, mais où celui-ci a le droit de parole $\left.{ }^{15} \gg\right)$; elle joue essentiellement une fonction rythmique, esthétique. Le tressage du dialogue opère une scansion, au sens de $\mathrm{Chion}^{16}$, du dit par le dit grâce aux différences de voix entre les locuteurs ou d'un même locuteur, par exemple lorsqu'une voix relance l'interlocution après une période narrative d'assoupissement mêlant la rêverie au ressouvenir. La conjugaison des voix peut faire succéder un mouvement de pensée monologique à un autre, cependant les mouvements de pensée se rejoignent. Introduite par un dialogue, la narration monologique sur le couvent restitue le temps long (une évolution sur presque deux millénaires); elle aboutit à une comparaison entre ses moines et le Christ abandonné. Un silence vient marquer la fin de cette période (au sens stylistique et mélodique du terme autant que simplement temporel). Un autre monologue débute, bien différenciable par la voix féminine qui l'énonce, mais reprenant le point d'arrivée du précédent. Une thèse se profile, sur la condition des plus défavorisés et la fraternité. Le mouvement de la pensée (au sens également musical du terme) n'est alors plus qu'à deux voix, et il se resserre sur une figure à la fois historique et symbolique, donnant son titre au premier volet du film, « Le Château ».

Cet énigmatique château est évoqué sur fond d'une série d'images d'une population maghrébine vivant dans la misère (voir la figure io). Sur l'image d'un garçon à l'air maussade, le monologue de la voix masculine reprend : «Eh bien, un jour j'ai vu des pauvres heureux. C'était à Nanterre au bidonville, premier jour d'indépendance algérienne. $\gg(24: 44-24$ :51 $)$

is Notion de Michel de Certeau résumée dans les termes de Charlotte BradySavignac dans « Pour une politique des voix au cinéma. À propos de "L'orgue et l'aspirateur", de Serge Daney », p.3, http://www.creationsonore.ca/wpcontent/uploads/20I8/II/Brady-Savignac_Daney_version_finale.pdf (consultation le 3o juin 2019); Michel de Certeau, « Le lieu et l'autre Montaigne: "des cannibales” », dans Maurice Olender (dir.), Le racisme. Mythes et sciences, Bruxelles, Complexe, i98I, p. I87-200.

${ }^{16}$ Michel Chion, 2006. La modalité de la scansion « correspon[d] au cas où ce que fait ou ne fait pas un personnage tout en parlant, ou encore un événement dans le décor sonore (son de klaxon, cri animal) ou le décor visuel (passage de voiture), ont pour effet de scander, de ponctuer le discours, et aident à le faire écouter par le spectateur $\gg$. 

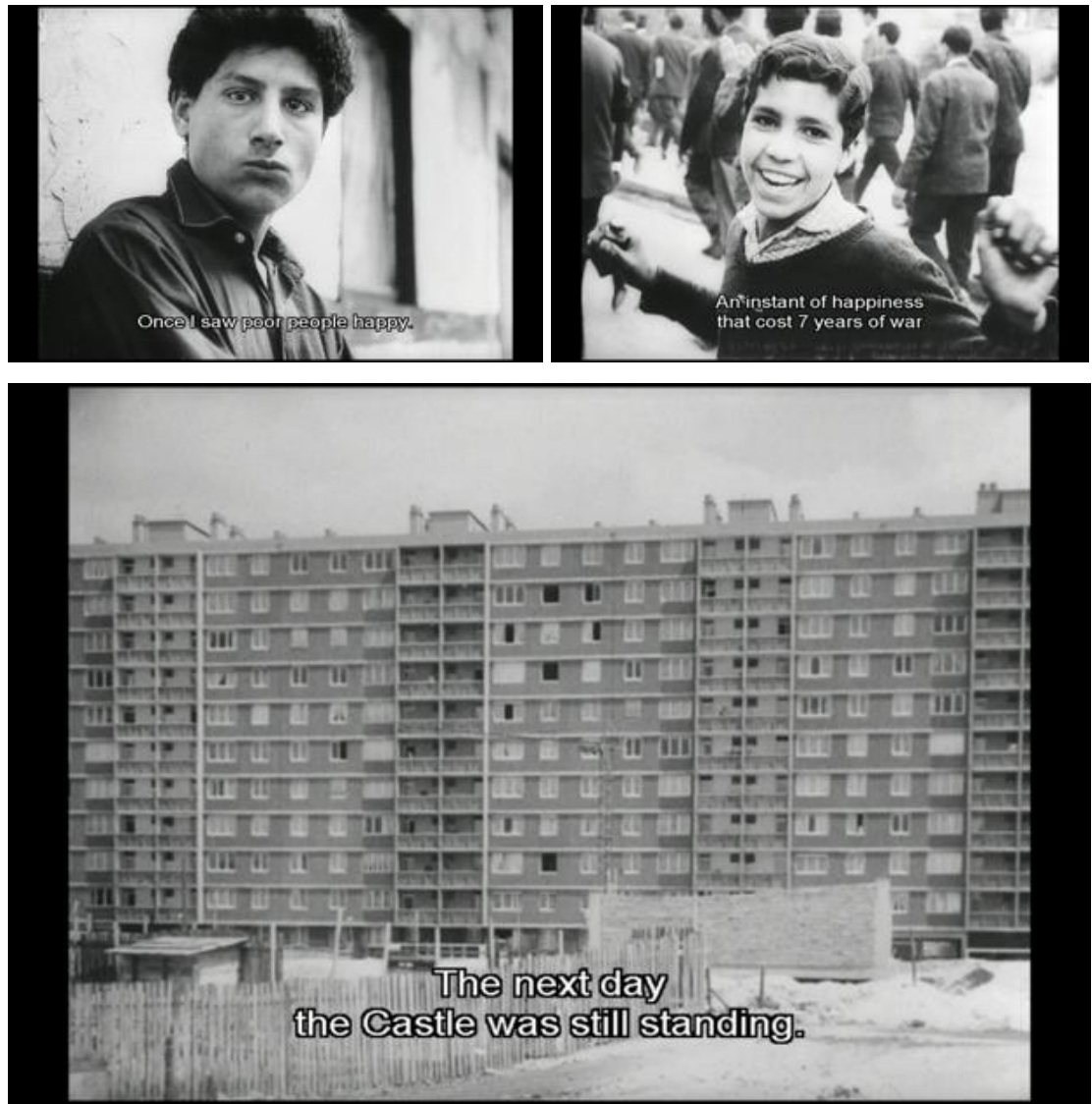

Fig. IO, II et I2 : Photogrammes du film Si j'avais quatre dromadaires, Chris Marker, 1966.

$\mathbf{I}_{19}$

À partir de ce contraste dit-montré ${ }^{17}$ s'engage une série d'images de cette population réjouie et triomphante (voir la figure II), pendant laquelle les voix des commentateurs se taisent, alors qu'on entend seulement un bruitage, musique concrète évoquant des actions violentes, tirs et coups de fouet, sur laquelle vient s'ajouter un lugubre air de violon (autre contraste, inverse).

Cette musique s'interrompt sur la photo d'un enfant radieux (voir la figure I2), et la voix féminine reprend : «Ils étaient beureux. Un instant de bonbeur payé par sept ans de guerre, et un million de morts. Et le lendemain, le château était toujours là. Et les pauvres sont toujours là, jour après jour. Et jour après jour, nous continuons de les trabir. » (25:37 - 26:II) Le signifiant « le Château », dont le sens

${ }_{17}$ Chion, 2006. 
était interprétable jusque-là de façon allégorique, s'associe alors à un référent précis, un immense bâtiment résidentiel « moderne » dans la banlieue pauvre de Nanterre, ayant pour nom ou surnom «le Château », sur lequel pèse une menace de destruction.

Les derniers commentaires de la voix féminine s'achèvent sur l'image d'un store de garage couvert de graffitis (voir la figure 13 ), dont l'un affirme : « Il ne faut pas démolir le château » (26:Io). On peut comprendre alors que « le Château », dans la composition du film, symbolise une antinomie - à la fois maintien de la pauvreté, des inégalités de classes, et foyer d'habitation, lieu de vie d'une communauté.

Il revient à la deuxième partie du film, « Le Jardin », de répondre à cette antinomie, à défaut de pouvoir la résoudre. La nouvelle série de photographies commentées est ainsi offerte comme le second volet d'un diptyque temporel. Ce volet revient sur l'idée d'éternité de façon désabusée, notamment à travers la séquence sur l'insatisfaction des Suédois, dont la société est présentée comme achèvement idéal du progrès humain :

- C'est bizarre, la mort, vous savez, quand on ne croit plus à la vie éternelle. [...] Leur bonbeur [aux Suédois] n'est pas suffisant pour équilibrer une absence éternelle. » (39:09-39:26)

À la suite de cette séquence et de celle sur la décrépitude du monastère du mont Athos, la notion d'éternité paraît désuète pour envisager l'avenir de l'humanité; il lui est préférée celle, plus modeste, de «survivance », avec cependant une note d'optimisme quant au progrès dans les relations interpersonnelles. « La loi du jardin », par opposition à celle de la jungle, nous amènerait, par accoutumance, à vivre mieux les uns avec les autres. Insérées dans cette séquence, des photographies de graffitis au ton désespéré sont en dialogue à la fois avec les graffitis concluant la première partie, et avec les voix (voir les figures I3 et I4). Celles-ci ménagent un recul critique par rapport au message de ceux-là; elles articulent en les incorporant — sans s'y laisser coller - le dernier mouvement de pensée. Le «j'aime personne » du dernier graffiti (voir la figure 14) est supplanté par une série de photographies montrant des gestes d'affection; les mouvements de caméra ciblent ces gestes, en valorisent l'importance tout en en faisant ressentir la ténuité. 
TEMPORALISER LA PHOTOGRAPHIE :

MARKER, VARDA, WENDERS-SALGADO
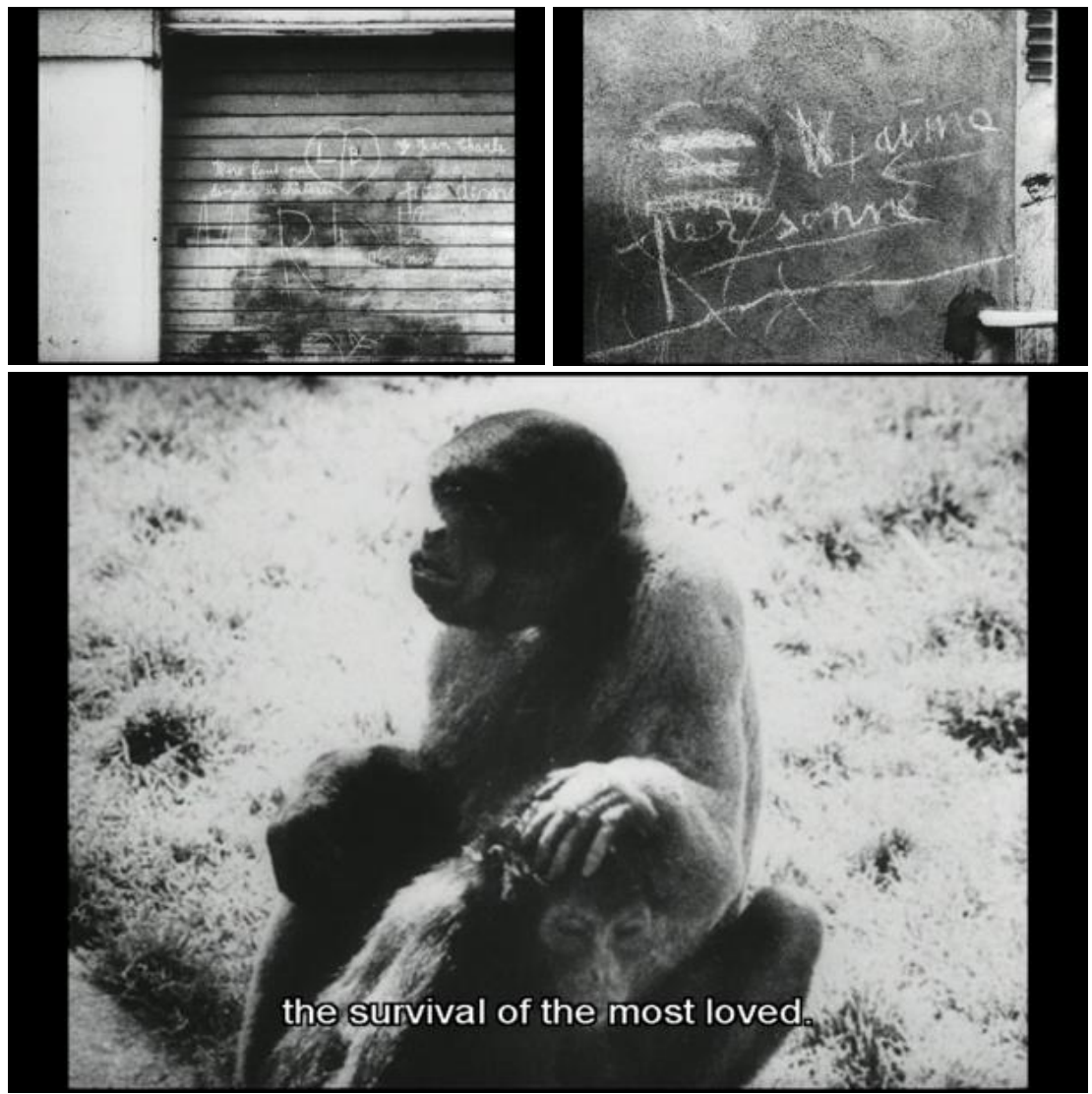

Fig. I3, I4 et I5: Photogrammes du film Si j’avais quatre dromadaires, Chris Marker, 1966.

Présentée comme nécessitant une subtile attention au monde, qui à première vue n'offre qu'un spectacle de violence, la découverte de ce «maquis», de cette « clandestinité du bonheur », «Sierra Maestra de la tendresse », est ponctuée par une dernière photographie où un macaque (voir la figure I4) pose affectueusement sa main sur la tête d'un autre. Celle-ci répond aux cris (acousmatisés) poussés par un singe, suggérant l'expérience du paroxysme de l'horreur, quelques instants plus tôt, par une série de photos montrant des affiches publicitaires et des slogans politiques gaullistes déchirés. Plus largement, cette dernière photographie, ou cette dernière série d'images associées au commentaire, conclut un propos qui pouvait encore paraitre décousu en lui donnant une cohérence d'ensemble: le thème de l'amour, en filigrane depuis les premiers propos sur l'acte de photographier, puis dans l'intérêt porté aux autres cultures et dans la dénonciation de l'oubli des déshérités, vient revigorer une vision spirituelle du monde où l'espoir en l'Éden et son éternité, n'ayant d'autre lieu que celui de l'art, a été laissé de côté. 


\section{ULYSSE : DES RETROUVAILLES À LA MYTHOGRAPHIE}

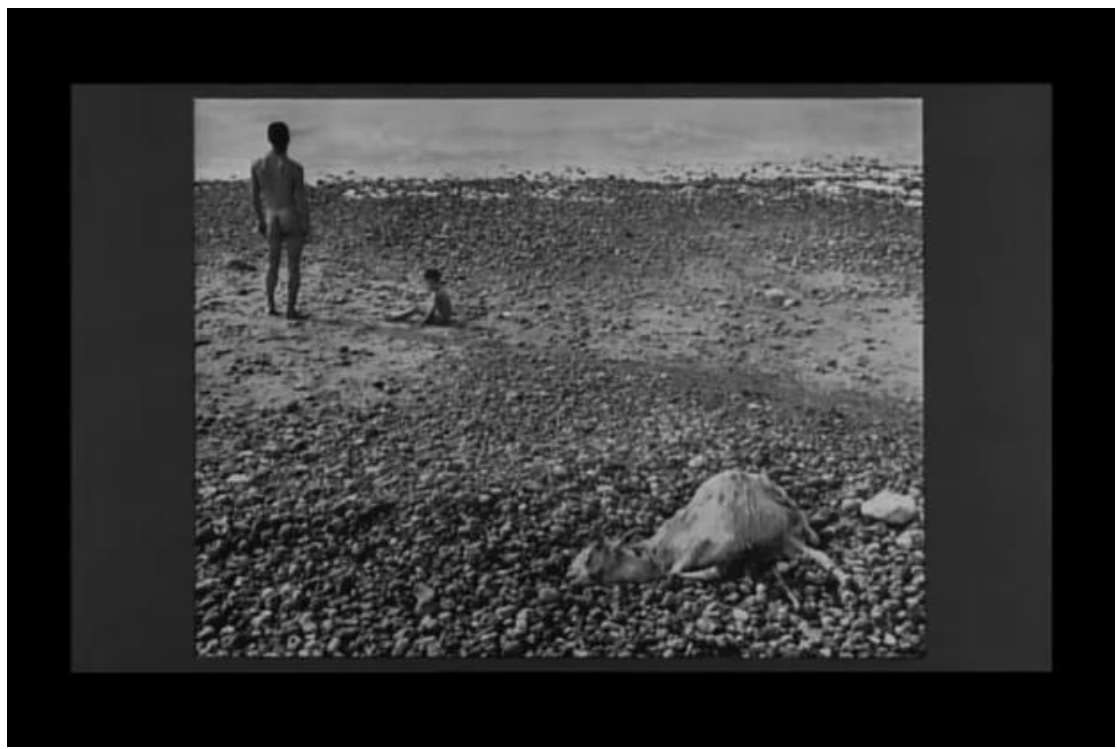

Fig. 16 : Photogramme du film Ulysse, Agnès Varda, 1982. Le court métrage Ulysse opère un retour exégétique sur une photographie prise par la réalisatrice vingt-huit ans plus tôt. Le travail commence par un effort de restitution du contexte biographique dans lequel la photographie a été prise.

«C'était un dimanche, sur la côte au bord de la Manche » : ainsi débute le propos de la narratrice acousmatisée, dont on peut reconnaître la voix, mais qui s'identifie («je ») tout de même comme étant à la fois l'auteure de la photographie et celle du film («Quand j'ai fait cette photographie [...] »). Une même auteure donc, qui parle à partir de cette photographie, prise 28 ans plus tôt, une même auteure à ceci près qu'un écart temporel se produit entre l'auteure de la première image et celle de l'œuvre cinématographique qui l'englobe et la déborde pour en pénétrer le mystère. La trace laissée par la photographie permet-elle de se relier à la présence d'autrefois ? Nous allons voir que le cheminement de Varda la réalisatrice multiplie les pistes de recherche qui se révèlent autant d'accès barrés au sens plein ou définitif.

La mémoire de l'auteure permet de restituer des éléments à la fois biographiques et techniques qui apportent quelque matière à interprétation : « [...] sur un appareil à plaque, j'ai vu l'image à l'envers sur le dépoli: l'homme planté par la tête en bas à droite, l'enfant centre-droit au lieu de centre-gauche, et la chèvre planant dans un ciel de météores comme un signe du zodiaque » (1:02 - I:22) (voir la figure 17). Ces informations sont saisies dans le récit de la narratrice-auteure : c'est elle 
qui manipule filmiquement la photographie pour reproduire la perception qu'elle en eut alors, et c'est elle également qui fournit l'interprétation donnant une dimension zodiacale au cadavre de la chèvre. Varda offre un commentaire restituant son cheminement de pensée créatif et inscrivant son geste dans la logique d'une tradition picturale :

- Et pourtant, je l'avais d'abord aperçue du baut de la falaise, couchée sagement, assommée sans doute par sa chute. Une chèure morte, c'était un sujet en or, un sujet de composition comme j'aimais les faire à l'époque. Nature morte ou paysage avec figures, comme disaient les peintres anciens. Des figures, c'est-à-dire des personnages nus dans la nature. Ici un enfant et un homme: mes modeles. (I:22 - I:50)

La narratrice présente sa photographie comme une œuvre, c'est-à-dire comme un artefact (une création) dont le motif n'est pas simplement de garder la trace d'un instant, mais de réaliser une composition artistique. Cependant, cela est loin de suffire à l'exégèse que se propose de faire l'artiste dans sa nouvelle œuvre (le film): commence alors un essai de restitution exhaustive du temps de la photographie. La stratégie de la cinéaste consiste d'abord à confronter ses souvenirs à ceux des personnages de son tableau.

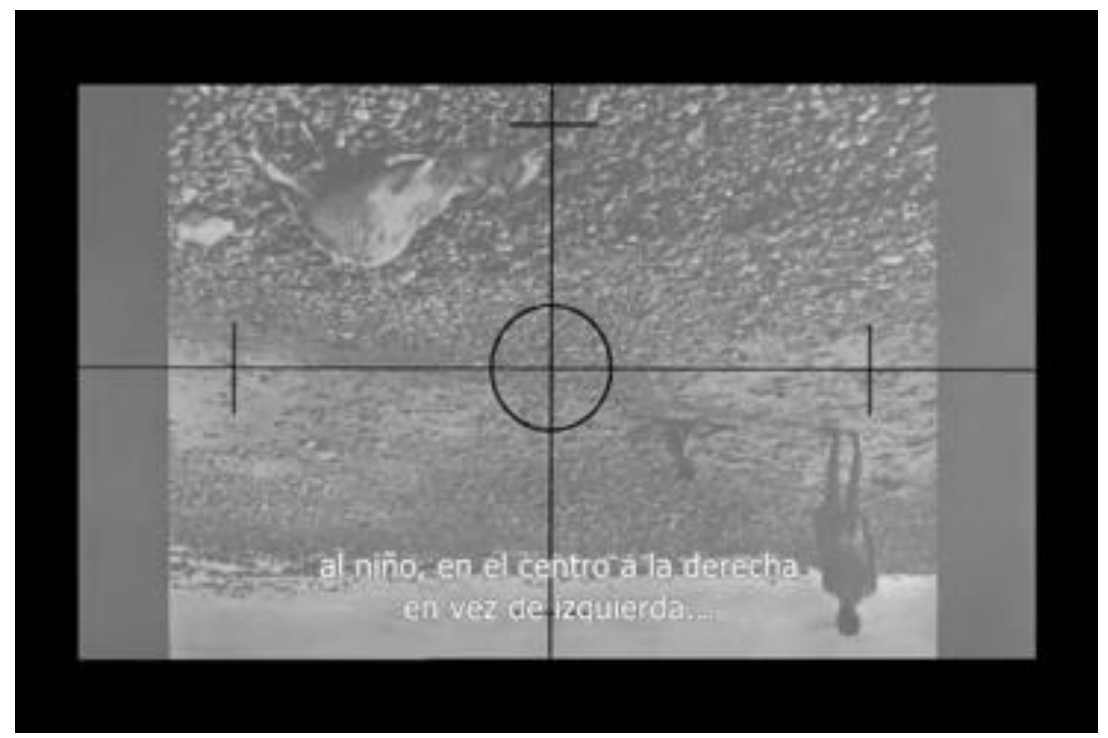

Fig. 17 : Photogramme du film Ulysse, Agnès Varda, 1982. 
Les personnages, hormis la chèvre, sont également des personnes vivantes au moment du tournage, ce qui devrait permettre de donner au film la tournure d'une exploration du passé de l'instant photographié à partir des vestiges mémoriels de ses acteurs. « Si la chèvre était née et morte à Saint-Aubin-sur-Mer, l'enfant était né à Dénia, et l'homme à Alexandrie. Il n'avait jamais vu cet enfant jusqu'à ce dimanche sur la plage. Et moi je n'ai pas revu l'Égyptien depuis ce jour-là. » (I:50 - 2:05)

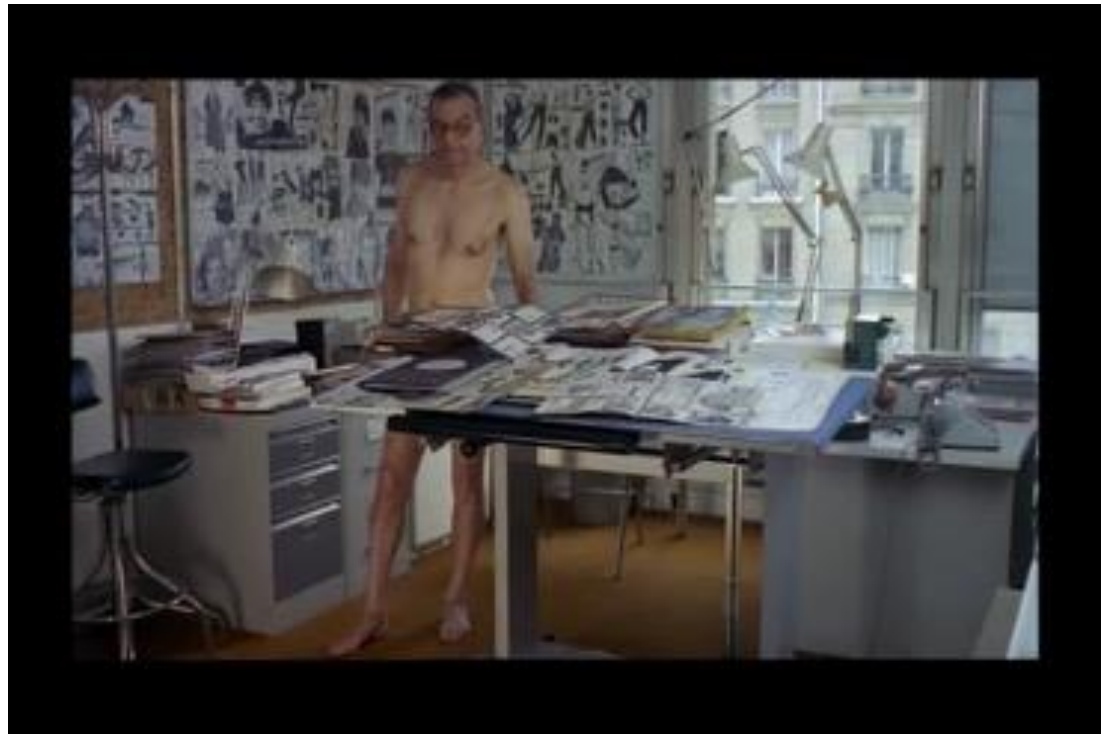

Fig. I8 : Photogramme du film Ulysse, Agnès Varda, 1982.

L'homme de la photographie était montré nu, debout, de dos, en noir et blanc; le film nous le présente également debout et tout aussi nu, mais partiellement (pudiquement) dissimulé, de face (voir la figure I8), en couleurs, et parlant, ou plus exactement dialoguant, avec la réalisatrice-narratrice : « Ça fait combien de temps? Ça fait combien de temps que je t'ai pas vue, toi? Ça fait 28 ans, 26 ans. Quelque chose comme ça, ouais, je crois que c'est ça. Depuis 55, 56, 54, je ne sais pas. », dit-il (2:052:25). La réalisatrice ne lui répond pas, le laissant dans l'incertitude — qui révèle la difficulté de se souvenir exactement, de restituer le temps écoulé. Ce n'est qu'après cette première introduction, faisant de la remémoration-restitution un problème, que la réalisatrice intervient en jouant les aide-mémoire au moyen d'objets (la photographie, des galets de la plage) et par un indice privilégié, le nom de l'enfant : 
- Je ne me souviens pas de celle-ci en particulier, [dit-il à propos de la photographie].

- Je me souviens bien du petit garçon.

- Tu te souviens de son nom?

- Euh...

- Il avait...

- Ouais, ça me paraissait un... oui...

- Ulysse.

- Oui, c'est ça, c'était pas Maurice ou...

- Non, c'est Ulysse... (2:40-3:02)

Les souvenirs qui reviennent à Fouli Elia n’ont pas trait directement à cette photographie. Mais ils continuent à faciliter une compréhension de l'approche artistique de Varda menant à celle-ci :

- Tu ne faisais pas de beaux paysages. C'était des...

- Des choses de rêveries, oui. (3:45 - 3:55)

Le film montre des photographies correspondant aux souvenirs qu'évoque Elia. La restitution du temps paraît ainsi reposer sur la capacité de remémoration de ceux qui en furent les témoins et acteurs. Cependant, l'essai photocinématographique en vient déjà à dépasser la simple tentative de restitution, en faisant de son itinérance à travers les difficultés mémorielles une manière de (com)poser artistiquement un problème philosophique : celui de la capacité à restituer (le temps) et de la pertinence d'un tel « geste » :

- Et ce portrait de toi habillé, tu te souviens?

- Non, ça je ne me souviens pas du tout, pas du tout. [...] Je ne me souviens même pas de cette veste, mais je me souviens de cette chemise. Je ne me souviens pas de cette personne-là [lui-même alors].

- Comme c'est troublant: on se souvient de ses habits, on ne se souvient pas de qui on était.

- Je ne veux pas, je ne veux pas me souvenir. Si on ne se souvient pas, on est mal parti. Je dis ça comme ça, qu'est-ce que je sais, moi? Eh bien, maintenant, puisque j'ai cent mille ans, je commence à comprendre.

- [Hors dialogue, s'adressant au spectateur la narratrice acousmatisée reprend cette idée:] Et moi qui en ai cinquante mille, je commence aussi, 
un peu. Mais est-ce que je sais ce que j'avais dans la tête il y a vingt-buit ans quand j'installais cet enfant au milieu de la plage? (4:08 - 5:16) retrouve ici ce que Barthes appelle dans $S / Z$ la gestion du code herméneutique ${ }^{18}$, c'està-dire du suspens(e) et du secret, à ceci près que le secret ou le mystère en est aussi un pour l'auteure, avant tout pour elle. Qu'est-ce que la narratrice et son interlocuteur commencent à comprendre ? Quelque chose qui ressemble à une promesse de sens est faite. Il nous est donné à nous, spectateurs, le sentiment que nous allons avancer dans la compréhension de ce mystère, qui en devient un pour nous, tout autant, voire plus désormais, que pour l'auteure-enquêteuse, qui paraît à la fois vouloir le percer (en tant que réalisatrice-actrice dans le présent filmé) et le contenir en elle (en tant que réalisatrice-narratrice ayant achevé le film).

Serait-ce en continuant à essayer de restituer l'autrefois de la photographie, ce temps perdu, qu'on pourrait parvenir à éclaircir son mystère, son obscure clarté ? Il s'agit d'une photographie où l'intensité lumineuse est forte, mais où le noir du noir et blanc, l'étrangeté de la scène - Qui sont ces personnages les uns pour les autres ? Que fait cette chèvre morte au premier plan ? etc. — créent une impression d'opacité. Varda l'auteure-narratrice du film renforce le mystère de la photographie à l'aide de paradoxes et d'oxymores implicites. Paradoxalement, c'est l'âge, le grand âge, le temps qui a passé depuis lors, qui donne un avantage, qui facilite l'élucidation. La vieillesse aide à comprendre le sens d'un autrefois devenu comme étranger à ceux-là mêmes qui l’ont vécu, qui en étaient les témoins et les acteurs. Mais la vieillesse est aussi ce qui épaissit le mystère de l'autrefois, le rend précieux, en fait une énigme — l'énigme d'un entrelacs d'existences en un point du temps, augmentée par un geste artistique qui compose le tableau et pérennise l'instant.

Commence alors l'exploration de la deuxième piste humaine, celle de «l'enfant», cet Ulysse dont la séquence précédente a ménagé le mystère. Un mouvement de caméra de gauche à droite sur la photographie, depuis l'enfant, est continué par un mouvement de caméra similaire sur un « réel » contemporain (au film) donnant à voir Ulysse aujourd'hui (habillé), accompagné par sa femme et ses enfants. Qu'apprend-on de cet Ulysse et de sa famille qui nous aide à élucider l'énigme photographique ? On apprend plus sur Ulysse que de cet Ulysse. Non pas qu'il refuse de se prêter au jeu de la remémoration, mais tout comme celle de Fouli Elia, sa mémoire est défaillante, pire encore, elle n'a gardé aucun souvenir de l'instant

\footnotetext{
${ }^{18}$ Roland Barthes, $S / Z$, Paris, Seuil, 1976, p. 25-27.
} 
sur la plage et de la photographie. Cela peut se comprendre dans la mesure où il était encore un enfant à l'époque. Cependant, que sa mémoire n'ait pas gardé de souvenir de cet instant et de cette photographie, cela est étonnant, comme l'indique Varda, parce qu'enfant il en avait fait une réinterprétation picturale. Varda la lui montre, mais rien n'y fait : l'Ulysse d'aujourd'hui n'a pas non plus le souvenir d'avoir fait cette peinture (voir la figure i9).

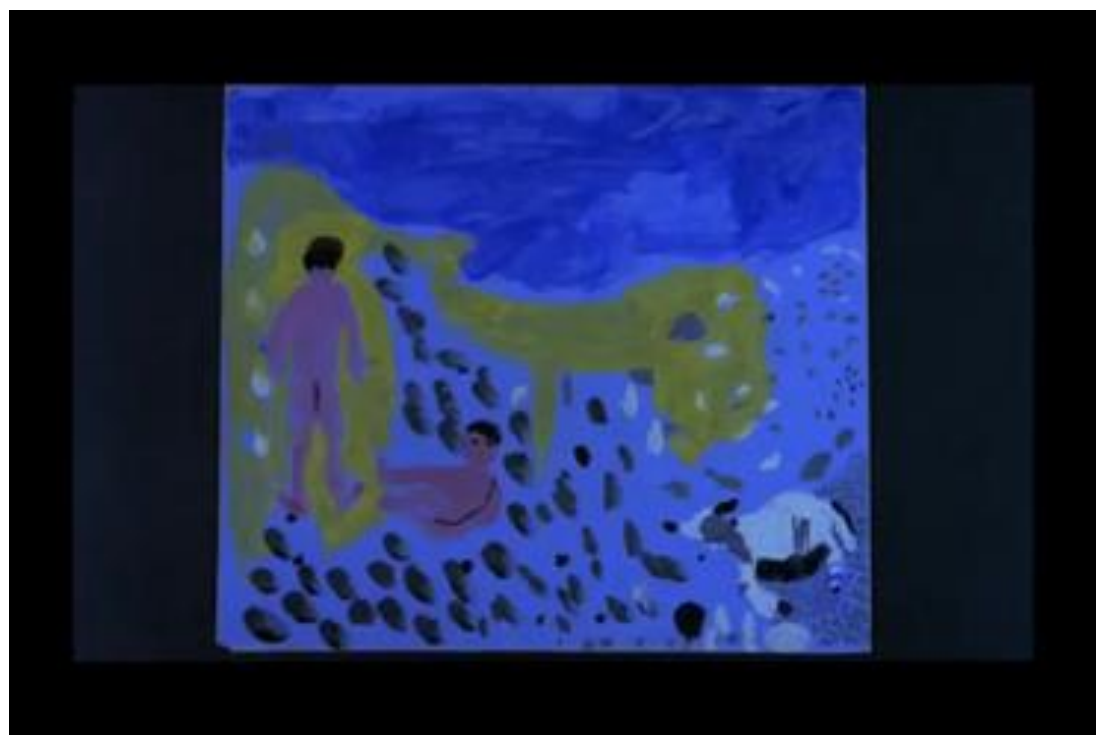

Fig. 19: Photogramme du film Ulysse, Agnès Varda, 1982. famille, son histoire, ses difficultés, et à sympathiser, à éprouver une sorte de familiarité compatissante, similaire à celle que paraît éprouver la réalisatrice. «Il a été mon premier enfant, mon premier enfant favori, mon enfant pour de rire. Car il avait une mère, Bienvenida, on disait Bienvé; et un père, Juan, maçon et républicain. Ils étaient espagnols bien sûr, et réfugiés politiques. [...]» (5:31 - 5:49) La photographie fonctionne alors davantage comme un prétexte à la narration de cette histoire familiale et amicale que cette dernière ne fonctionne comme la restitution d'un contexte qui permettrait de comprendre la photographie. Les faits biographiques mentionnés paraissent en effet rester anecdotiques, sans pertinence par rapport à la composition de la photographie, comme le soulignera Varda à la fin du film. Ce ne sont pas en tout cas les traces mémorielles d'Ulysse adulte qui apportent un contenu informatif profitable à son interprétation. Mais cette absence de souvenir quant au moment et à la photographie, 
l'échec de l'anamnèse, apporte davantage matière à réflexion qu'un simple ressouvenir. Le fait que le questionnement de Varda ne parvienne pas à réactiver ce souvenir, malgré la part active de l'enfant dans leur transformation en souvenir — au sens ici également matériel du terme, au sens de ce que Stiegler appelle une rétention tertiaire ${ }^{19}$ - accuse l'obscurité du problème, renforce le mystère. Si le témoignage d'Ulysse adulte ne joue pas un rôle d'adjuvant dans l'élucidation de la photographie, il n'en est pas moins précieux en ce qu'il fortifie la légitimité du projet de la réalisatrice, en accroît le sens philosophique.

Après ce double échec de la mémoire humaine, serait-ce alors, contre toute attente, la chèvre qui pourrait venir en aide ? C'est la piste alors explorée, qui présente une difficulté de méthode: comment interroger une chèvre qui était déjà morte au moment de la photo (voir la figure 20) ? Il suffit de passer de cette chèvre à « n'importe quelle » chèvre. À la suite d'une série de propositions négatives, profitant au passage de la valeur de prestige que donnent à son propos les artefacts culturels qu'elle passe en revue, Varda en vient à dégager une suggestion, une hypothèse, qui ne semble pas procurer davantage qu'un jeu de mots et d'images: «Puis-je suggérer qu'il existe un imanginaire ${ }^{20}$ animal? Un imaginaire autoprédateur?» (Iо:48 - II:46)

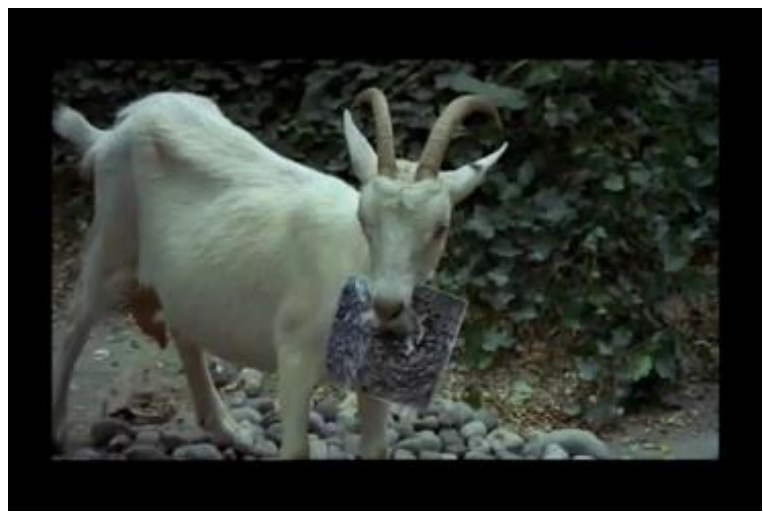

Fig. 20 : Photogramme du film Ulysse, Agnès Varda, 1982.

19 Les rétentions tertiaires, «[c]e sont les sédimentations (conscientes et inconscientes) qui se sont accumulées au cours des générations, et qui constituent de ce fait un processus d'individuation collective $\gg$. Définition disponible sur le site Ars Industrialis, créé par Bernard Stiegler en 20I5, http://arsindustrialis.org/r\%C3\%Agtention (consultation le 30 juin 2019); Bernard Stiegler, La technique et le temps, Paris, Galilée, coll. « La philosophie en effet », 2018.

${ }^{20}$ Mot-valise créé par Varda. 
Cette hypothèse n'est pas gratuite. Elle advient comme une façon créative de revenir sur le problème découvert précédemment, celui du défaut de remémorationrestitution, expliquant, de façon artistique et encore énigmatique, la déperdition des traces de mémoire.

Une méthode similaire à celle appliquée à la chèvre est alors appliquée à l'enfant. Puisque le garçon de la photo ne peut plus répondre ni du moment, ni de la photo, ni de sa propre peinture, interrogeons n'importe quel garçon, une ribambelle de garçons sur ces deux œuvres. Peut-on restituer par d'autres le sens d'œuvres qui sont devenues, au cours du temps, problématiques, voire étrangères à leurs propres acteurs ? La naïveté - la nouveauté du regard porté et la jeunesse des regardants peut-elle venir au secours d'une mémoire défectueuse face à un objet opacifié ? Cette hypothèse différencie notablement l'approche des précédentes : il s'agit d'essayer une interprétation quasi pure, hors mémoire et dans une certaine mesure hors culture, tenant pour rien la restitution du temps.
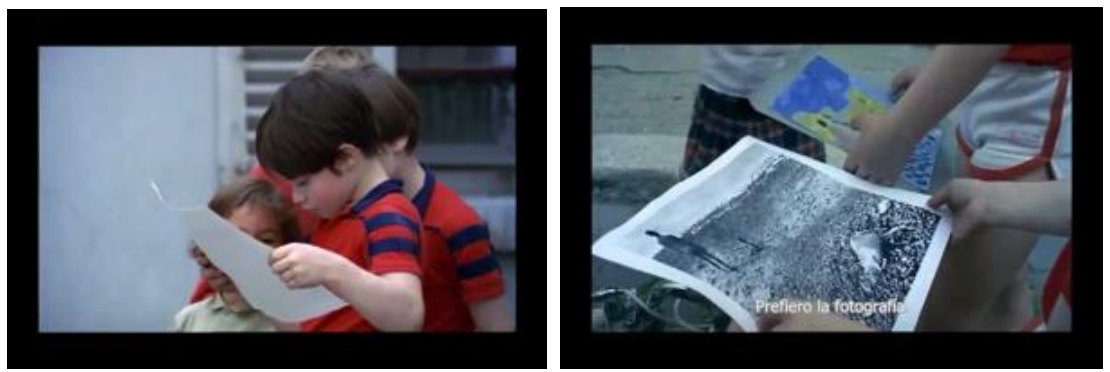

Fig. 2I et 22 : Photogrammes du film Ulysse, Agnès Varda, 1982.

Il est manifeste que cette piste ne constitue pas pour Varda un aboutissement, mais seulement un moment dans le processus exégétique, puisqu'elle en vient à emprunter la démarche opposée, à savoir interroger la mémoire collective, à travers une autre rétention tertiaire, celle constituée par les nouvelles dans la presse et à la télévision: «Les enfants disent: "bumain, vrai, réel". Qu'est-ce qui était réel ce jourlà pendant que j'étais à la plage? Que se passait-il ce 9 mai I954? 》 (13:35 - 13:47)

En abandonnant le biographique au profit de l'historiographique et en externalisant la mémoire, le recours aux archives nationales redonne-t-il une pertinence à la tentative de restitution du temps pour l'interprétation de la photographie? Varda explicite sa démarche: «Ce que j'en dis, ce n'est pas de mémoire. J'ai dîu fouiller le sable de la plage, et les actualités filmées, et les journaux de ce jour-là. » (I5:II - I5:I7) L'effort de restitution par remédiation est fait dans un 
style qui mêle l'humour critique à l'objectivisme historiographique : « La conférence de Genève venait de s'ouvrir. Pham Van Dong y représentait le Viêt Minh, et passait sans doute son dimanche à regarder le plus neutre des jets d'eau, pendant que les Français jetaient des bombes sur le delta du Tonkin. » (I5:18 - I5:31) Cette tentative de restitution est évaluée dans une interprétation philosophique qui en minimise l'importance quant à la lecture de la photographie : « Quand on regarde le bord de l'eau, le temps n'est plus le même. Les acteurs de l'Histoire, ceux qui font la mémoire officielle, passent comme les ombres vues depuis la caverne de Platon. 》 (16:12 - I6:19) (voir les figures 23 et 24 ).
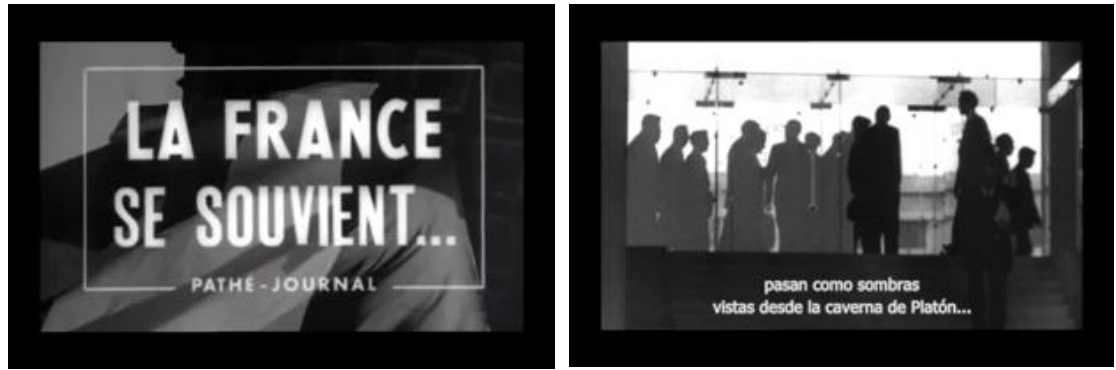

Fig. 23 et 24 : Photogrammes du film Ulysse, Agnès Varda, 1982.

De nouveau, la photographie - l'expérience qu'en a eue Varda lorsqu'elle l'a prise et la perception qu'elle en a au moment du film — fait tomber à l'eau la tentative d'explication contextuelle. Tournant cette découverte à son avantage, Varda revendique l'inactualité de l'œuvre et son autonomie d'artiste par rapport aux différents événements contemporains. Cette revendication s'appuie sur un certain idéalisme créatif :

- Sagesse ou égoïsme, moi, j'étais toute à l'image de l'instant, aux images que je voulais exposer quelques semaines après. [Elle mentionne et montre plusieurs photographies.] Et bien sûr, la photo d'Ulysse et compagnie, faite ce jour-là où je poursuivais une idée, une idée d'image. (16:28 - 17:06)

L'idéalisme, même créatif (comme Nietzsche, Bergson ou encore Derrida nous en préviennent), a tendance à tenir pour nulle l'importance du temps. Après cette enquête rétrospective polymorphe, Varda en vient à tirer une conclusion qui 
annule la valeur exégétique de celle-ci au profit d'une hypothétique ouverture totale de l'interprétation ${ }^{21}$ :

- Et voilà, j'ai situé cette image dans ma vie et dans son époque, comme on disait de le faire à l'école, mais les anecdotes, les interprétations, les bistoires, rien n'apparaît dans cette image. J'aurais pu la faire dimanche dernier ou bier, moi ou quelqu'un d'autre. L'image est là, c'est tout. Une image, on $y$ voit ce qu'on veut. Une image, c'est ça, et le reste. (17:47 - I8:09)

Le film a certes eu pour effet de minimiser l'importance de la restitution du contexte bio-historiographique de l'œuvre pour ce qui est de son interprétation. Mais n'a-t-il pas eu aussi pour effet - contrairement à ce que sa conclusion affirme - de limiter l'interprétation ? Une personne ayant vu le film voit moins en la photographie ce qu'elle «veut » qu'une personne n'ayant vu que la photographie. Participent désormais à son interprétation toutes les informations dont elle se souvient. Même si l'auteure du film et de la photographie l'autorise à interpréter celle-ci librement, le film lui-même informe son interprétation. Cette personne a connaissance du concours de circonstances qui a permis la rencontre des acteurs de la photographie, de la démarche artistique de l'auteure, du contexte biographique et sociopolitique ainsi que des interprétations que l'auteure se fait de sa propre œuvre, et sa perception de la photographie en est nourrie. La photographie vue par cette personne est désormais davantage qu'une image, elle est un instant d'histoire, un condensé d'histoire(s). Toutefois, si le film referme l'énigme de la photographie sur le nom d'Ulysse doublement éponyme, le rapport entre les deux œuvres (la photographie et le film) multiplie les référents. Ulysse renvoie au petit garçon devenu grand, à la photographie, au court-métrage, au personnage mythologique, et les donne pourtant à penser comme formant une unité, un seul signifié mystérieux. L'intermédialité du signifiant film rend ainsi sensible l'incapacité à restituer un temps qui donnerait tout son sens à l'œuvre photographique, et cet « échec » en garantit l'ouverture.

\footnotetext{
${ }^{21}$ C'est la conclusion que partage Ari J. Blatt dans son article, qui offre de plus une profonde interprétation du lien entre photographie et cinéma en comprenant l'œuvre de Varda comme une méditation sur l'historicité de ces arts visuels. « Thinking Photography in Film, or The Suspended Cinema of Agnès Varda and Jean Eustache », French Forum, vol. 36, $\mathrm{n}^{\mathrm{os}} 2 / 3$, 20II, p. I8I-200.
} 


\section{LE SEL DE LA TERRE : DU TEMPS DE LA SINGULARITÉ À CELUI DE LA TERRE}

Le film de Wim Wenders et de Juliano Ribeiro Salgado sur Sebastião Salgado est principalement un film bio-historiographique. Cet adjectif composé convient particulièrement pour décrire cette œuvre ou ce type d'œuvres, qui, à travers le portrait d'un être humain, retrace des moments historiques. Le biographique devient un mode d'exploration de l'histoire, et les moments de l'histoire compose la biographie. La restitution de l'histoire personnelle du « héros » ne rétrécit pas le champ du regard, elle est donnée au contraire comme ce qui permet d'ouvrir le regard du spectateur à un vaste horizon. Les anecdotes biographiques sur l'homme sont présentées comme un acheminement vers une meilleure compréhension de l'Homme.

- [Sebastião Salgado père :] Ce chenapan n'aimait pas l'école, il nous a donné du fil à retordre. Mais il a étudié l'économie. J'aurais voulu qu'il soit avocat. Son année de droit ne lui a pas plu. Alors, il a fait économie et il a réussi.

— [Wim Wenders :] C'était Sebastião Salgado, le père. Il a transmis son nom à son fils unique. Celui-ci, même s'il est resté un grand voyageur toute sa vie, a profité des études que son père lui avait imposées, d'une manière que lui-même n'aurait jamais imaginée. Ces études d'économie lui ont permis de comprendre les marchés mondiaux, le commerce et l'industrie. Il savait donc ce qui faisait tourner le monde. Tout a commencé dans la petite ville d'Amores, au cour du Brésil. [...] (12:54 - 13:54)

Ces informations biographiques sur Sebastião Salgado (fils) ont fréquemment pour fonction de légitimer son interprétation des événements, et de les faire accepter comme de simples explications. Et, en effet, les longues périodes qu'il a passées au sein des communautés qu'il a photographiées, son regard d'économiste-ethnographe, son travail de photographe mué par un amour de l'humanité (interprétation prééminente de Wenders, qui explique le titre métaphorique du film), font de lui un observateurnarrateur auquel il paraît peu probable qu'un spectateur ne veuille pas accorder $\mathrm{du}$ 
crédit $^{22}$. Sa narration, commentant les photographies qu'il a lui-même prises, se donne comme une pure restitution des moments historiques dont il a été témoin.

Cependant, le discours du photographe-commentateur va souvent, pour ne pas dire toujours, au-delà de ce travail de restitution du contexte biohistoriographique. Il va même jusqu'à donner aux images commentées une valeur qui paraît aller à l'encontre de leur historicité, une valeur mythique ou panhistorique (qui vaudrait pour toute l'histoire de l'humanité), voire anhistorique ${ }^{23}$. Sans surprise, le discours panhistorique commente des photographies panoramiques :

- La Serra Pelada, la mine d'or du Brésil, en face de moi. Quand j'arrivais au bord de cet immense trou, tous les poils de mon corps montaient. J'ai jamais vu une situation pareille. Là, j'ai senti déroulée devant moi, [en] quelques fractions de seconde, l'histoire de l'bumanité: l'histoire des constructions des pyramides, la tour de Babel, les mines du Roi Salomon. On n'écoutait [entendait] pas le son d'une seule machine là-dedans. On écoutait seulement les murmures de cinquante mille personnes dans un grand trou: les conversations, les bruits, les mouvements, mélangés avec cette touche manuelle. Vraiment, je suis venu au début du temps, là. Je sentais presque le murmure de l'or dans l'âme de tous ces gens. (oo:45 - oI:43)

À ces moments d'interprétation panhistorique succèdent des phases de commentaires correspondant à un regard plus rapproché. Ces commentaires concourent à produire l'impression de cheminer parmi les personnes photographiées. Ils accompagnent le mouvement de séquences photographiques qui invitent à s'immerger dans la culture

${ }^{22}$ Nous ne voulons pas dire que les critiques défavorables au travail du photographe n'existent pas. On note en particulier l'existence d'un article académique récent particulièrement réprobateur : «Sebastião Salgado, ou a lógica enervante do colonialismo tardio », Igarapé, vol. II, n ${ }^{\circ}$, 20I8, p. 20-36. On peut mentionner aussi celle de Francisco Cubas, «Wenders filma un gran promocional para Salgado », El Fotografo Lector, 2015, en ligne, https://elfotografolector.com/2015/06/or/wenders-filma-un-gran-promocionalpara-salgado/ (consultation le zo juin 2019), qui porte sur le film lui-même et dénonce en celui-ci une hagiographie promotionnelle. Pour une discussion des critiques précédant le film, voir Pavarti Nair, A Different Light: The Photography of Sebastião Salgado, Durham, Duke University Press, 20II.

${ }^{23}$ «Qui ne tient pas compte du point de vue historique. » (définition du dictionnaire Larousse). 
de certaines peuplades isolées, à éprouver certaines situations (souvent catastrophiques) du dedans. Dans la séquence de la Serra Pelada, les hommes qui apparaissaient d'abord comme des fourmis deviennent des visages, des expressions, des gestes, des muscles tendus, des histoires (voir la figure 25).

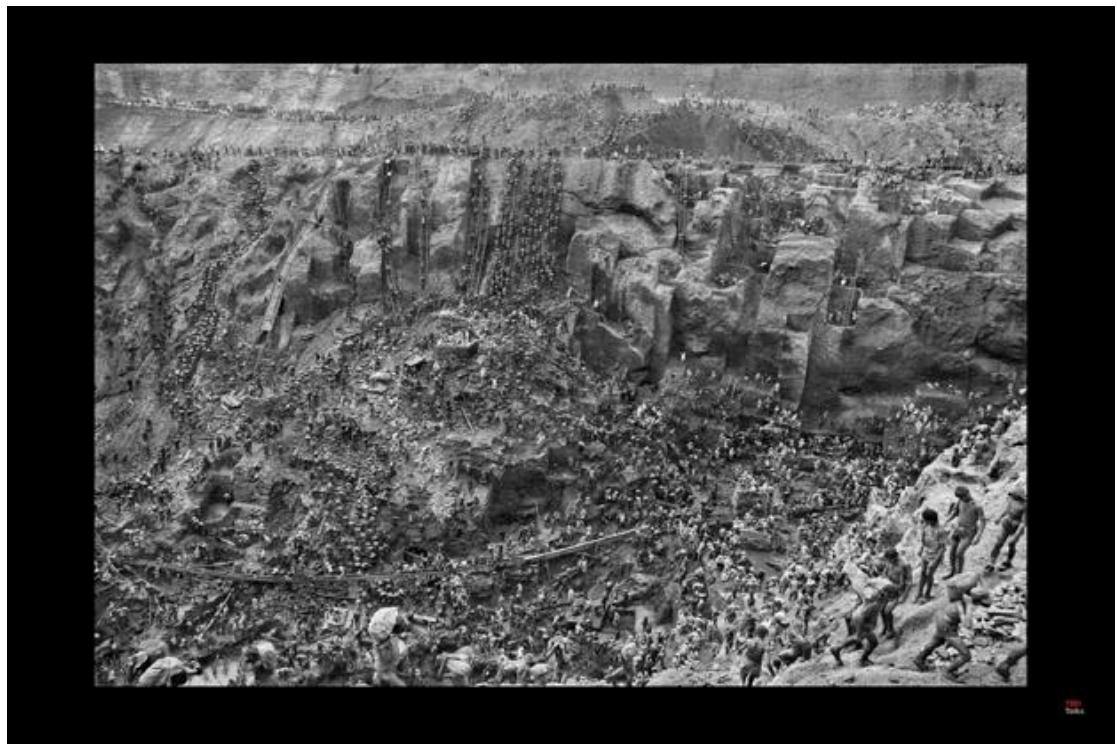

Fig. 25 : Photogramme du film Le sel de la Terre, Wim Wenders et Juliano Ribeiro Salgado, 2014 .

Quelquefois les commentaires de Sebastião Salgado convient à prêter attention à un instant précis, ayant eu une forte portée affective pour lui. La narration marque des pauses, détaille tels cas, en même temps que certaines photographies font ressortir la singularité de telles personnes, de tels moments :

- C'est un Tarabumara, avec son visage bien marqué, bien vécu. Des beaux cheveux, hein? Des cheveux fantastiques. Plusieurs fois, j'ai eu les gens qui venaient vers ma caméra, j'avais l'impression que j'étais plutôt un preneur de son. Ils me racontaient des choses comme si j'étais en train d'enregistrer ce quils me racontaient. $(25: 30-25: 55)$ 
On peut noter ici comment le commentaire passe du mode singulatif au mode itératif $^{24}$ : la musique hors champ, celle d'un air de violon, la réitération de la même image après une coupure montrant Salgado la commentant, opèrent la transition entre ce violoniste et l'idée de capter les sons. On observe ainsi la tendance du commentaire à relier l'expérience d'une singularité à celle d'un groupe de personnes — pouvant appartenir à différents groupes ethniques.

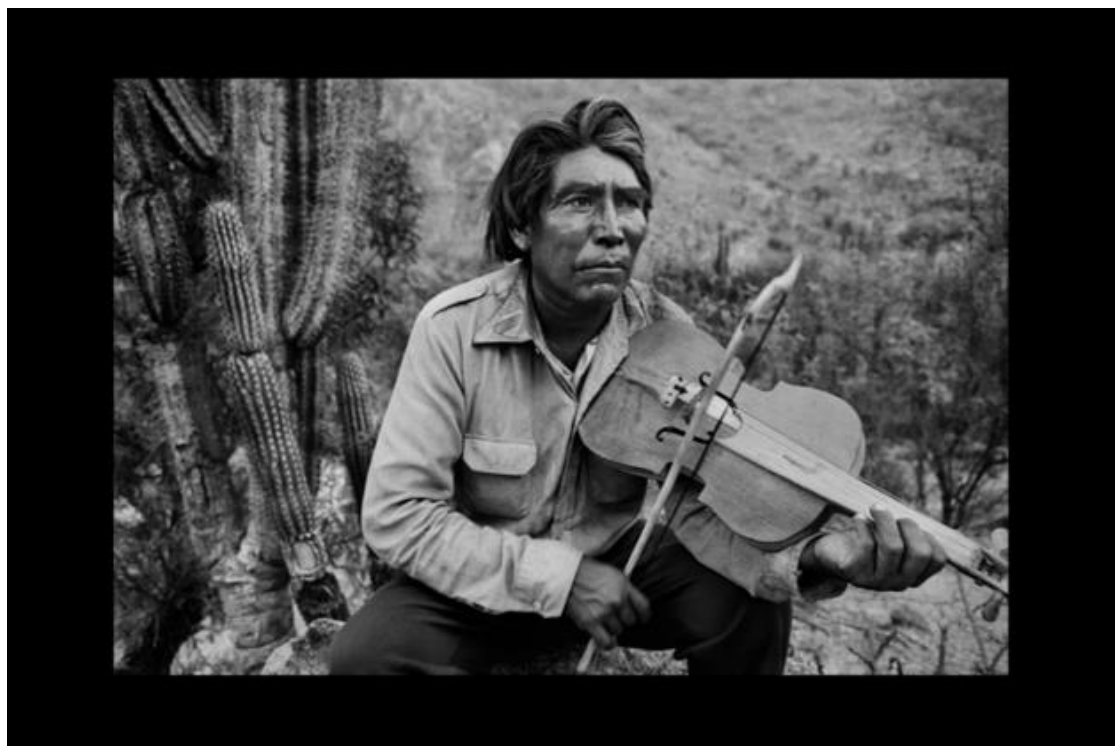

Fig. 26 : Photogramme du film Le sel de la Terre, Wim Wenders et Juliano Ribeiro Salgado, 2014 .

À l'échelle ethnographique se précise un aspect paradoxal et cependant essentiel de la restitution du temps dans le travail photographique : rendre sensible à travers une ouvre sans durée la différence qualitative de la temporalité propre à chaque groupe ou situation. Pour Salgado, il ne s'agit pas seulement de donner les informations permettant de comprendre le contexte sociohistorique, mais, par la restitution de ses impressions de voyage dans son commentaire, de sensibiliser à cet

${ }^{24}$ Pour ces concepts narratologiques bien connus, voir Gérard Genette, « Fréquence », Figures III, Paris, Gallimard, p. I45-182. Le singulatif désigne « cette forme de récit où la singularité de l'énoncé narratif répond à la singularité de l'évènement narré », p. 146; le mode itératif, par opposition, désigne «ce type de récit où une seule émission narrative assume ensemble plusieurs occurrences du même évènement (c'est-à-dire, encore une fois, plusieurs évènements considérés dans leur seule analogie »), p. I48. 
aspect qualitatif, et ce, sans avoir à parler directement de son propre travail artistique pour l'exprimer :

- J'ai peut-être jamais rencontré dans ma vie tout un peuple qui avait un autre rythme du temps. Le temps que je passais avec les Saraguros... j'avais l'impression que je passais une centaine d'années avec eux, tellement tout était lent, était dans une autre manière de penser, une autre vitesse. Il y avait un fatalisme sur leurs visages. (23:27 - 23:50)

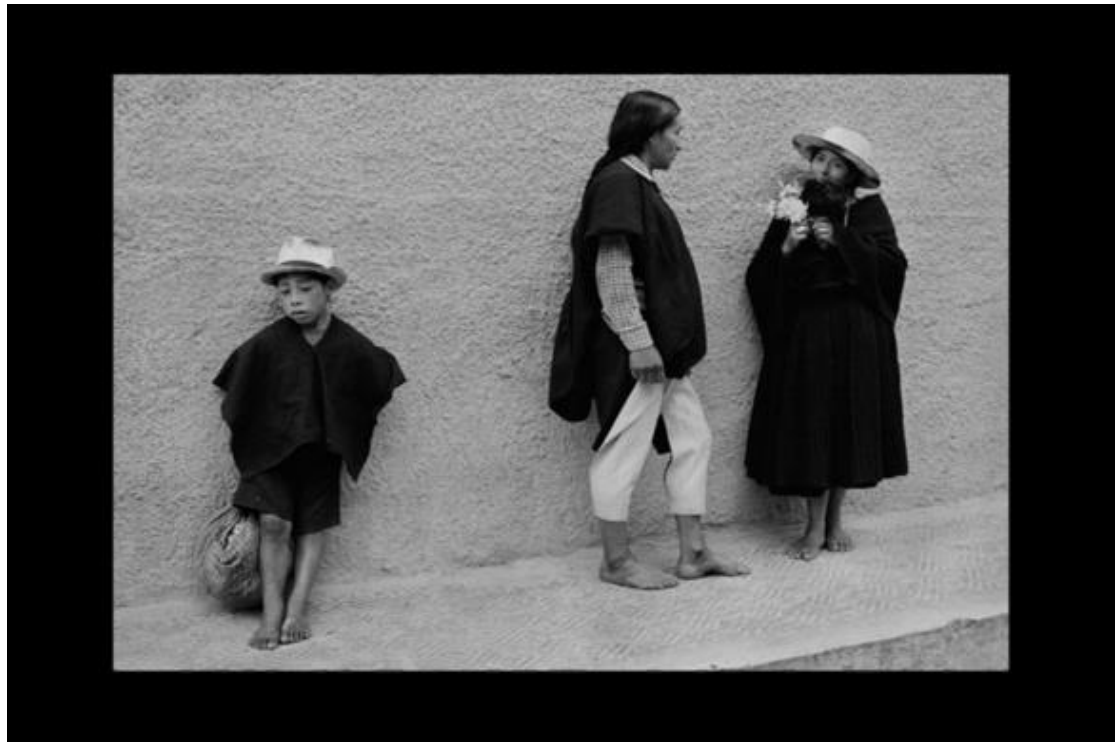

Fig. 27 : Photogramme du film Le sel de la Terre, Wim Wenders et Juliano Ribeiro Salgado, 2014 .

Comme l'explique Husserl dans ses Leçons pour une phénoménologie de la conscience intime $d u$ temps, « [d] urée de la sensation et sensation de la durée font deux $25 \gg$. Ce n'est pas en maintenant à l'écran les images des Saraguros plus longtemps (que les autres images) qu'une sensation de lenteur est produite, mais bien grâce à la paradoxale captation d'un rythme de vie par la photographie, dans son instantanéité. Le commentaire ne fait ici que rendre plus évidente cette sensation - et le fait que la rendre sensible faisait bien partie d'une intentionnalité artistique.

${ }^{25}$ Edmund Husserl, Leçons pour une phénoménologie de la conscience intime du temps, trad. J.-F. Pestureau, Grenoble, Jérôme Millon, coll. « Krisis », 2003, p. 2 I. 
Wim Wenders dévoile son attachement personnel à certaines photographies, en particulier à celle, fameuse, d'une femme touareg aveugle (voir la figure 28), et il raconte comment sa rencontre avec le photographe s'est faite à partir de cette première rencontre avec deux ouvres spécifiques : «J'ai découvert cette photo dans une galerie il $y$ a plus de vingt ans. [... $] \gg(4: 35$ - 5:24). Cette restitution du contexte qui a amené le réalisateur à découvrir le photographe retrace la genèse du film, sa préhistoire; c'est également une manière de valoriser la réception avec sa part de hasard et de prédétermination (l'inoculation d'un intérêt, au sens fort du terme, qui, longtemps plus tard, lui fera consacrer trois ans de travail au photographe). Ainsi, la restitution du temps de la réception indique l'importance de la singularité - de la personne photographiée et de l'instant où telle expression, dans son intensité, a été saisie.

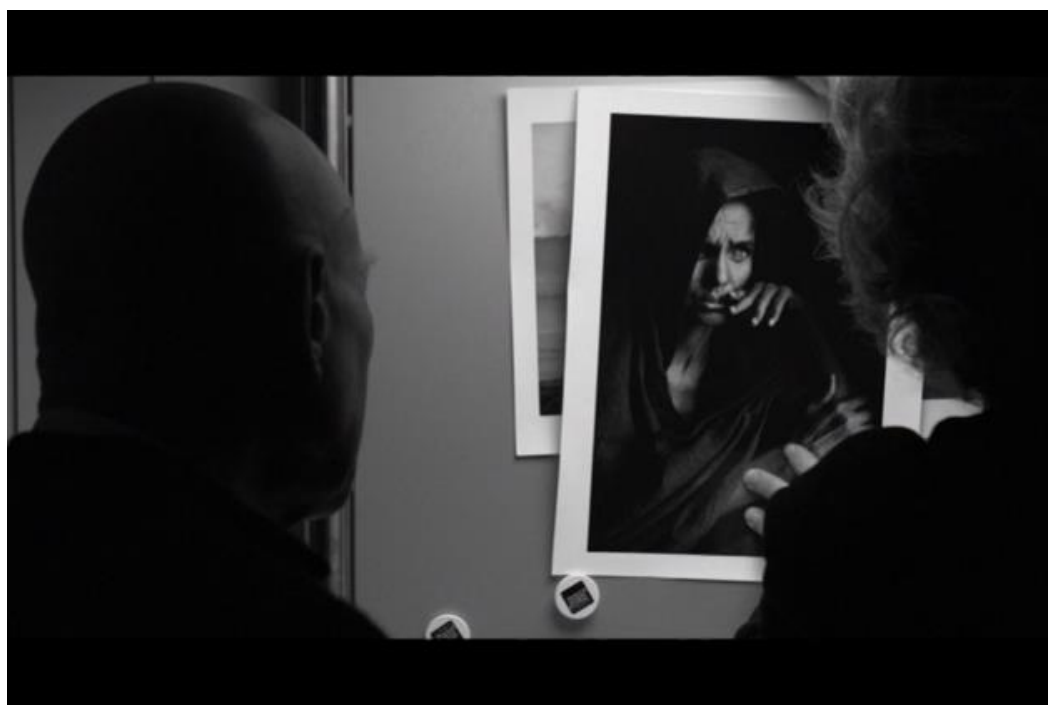

Fig. 28 : Photogramme du film Le sel de la Terre, Wim Wenders et Juliano Ribeiro Salgado, 20I4.

Néanmoins, le propos général du film et du photographe lui-même tend à donner une dimension archétypale aux personnes photographiées. Le commentaire tend à généraliser l'action décrite, à la décrypter en effet du point de vue d'un économiste-ethnographe-humaniste. L'anecdotique s'efface au profit d'une vision d'ensemble. L'auteur-commentateur incline à percevoir le temps que restituent les photographies comme un temps exemplaire. On peut déceler ici une intentionnalité auctoriale double et presque contradictoire : témoigner de situations extraordinaires, d'individus ou de groupes singuliers, et rendre compte d'expériences qui révèlent 
l'humanité dans son ensemble. La contradiction tient sans doute à la manière même dont Salgado a travaillé et au dispositif filmique : en passant de longues périodes de temps parmi les personnes qu'il photographiait, il se disposait à recueillir l'insolite; en élaborant des albums à partir de ses photographies, et plus encore en participant à un film qui retrace la trajectoire de tous ses albums, Salgado et les réalisateurs énoncent une parole qui dessine à grands traits, qui trace à partir d'une immense archive une figure compréhensible.

On trouve dans Le sel de la Terre des réflexions sur la photographie rappelant, à certains égards, celles que proposaient Chris Marker ou Agnès Varda :

- La force du portrait, c'est que, dans cette fraction de seconde, on comprend un peu la vie de la personne qu'on photographie. Les yeux racontent beaucoup, comme l'expression du visage. Quand tu fais un portrait, c'est pas toi tout seul qui prends la photo. La personne t'offre la photo. $(26: 00-26: 25)$

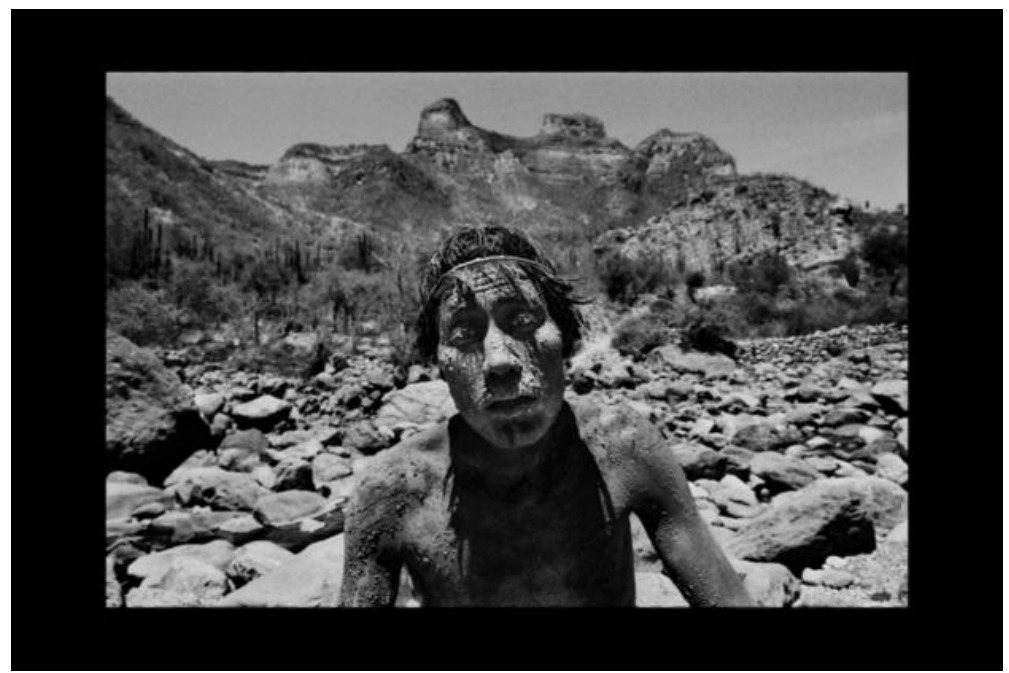

Fig. 29: Photogramme du film Le sel de la Terre, Wim Wenders et Juliano Ribeiro Salgado, 20I4.

Contrairement aux deux films précédents, celui-ci ne construit pas à partir ou autour du travail photographique un mystère à interpréter. Dans ce film, les cinéastes autant que le photographe travaillent à expliciter le sens, celui de presque chaque image et celui de l'œuvre en son entier à travers son évolution. L'œuvre ne se constitue ni en oracle ni en image infiniment interprétable. Elle témoigne d'une « éternité » 
qui se situe hors d'elle - celle de la nature. À la fin du film, en lien avec la dernière période du travail de Salgado, la restitution du temps prend un tout autre sens : il ne s'agit plus de restituer un contexte bio-historiographique, mais les conditions biologiques de la Terre avant les désastres causés par l'humanité. Le dispositif photographie-cinéma aboutit à une sortie de l'œuvre du domaine de l'art (au sens restreint du terme) : l'œuvre devient écologique, et le film comme la photographie, des moyens d'y sensibiliser. Restituer le temps suivant le projet Instituto Terra de Lélia $^{26}$ et Sebastião Salgado consiste à réparer notre planète, à restaurer son état virginal, et donc paradoxalement et, dans une certaine mesure, utopiquement, à restituer un temps anhistorique, si l'on entend par histoire, comme habituellement, celle d'une humanité transformant à grande échelle la nature. Le titre du dernier album des Salgado est à cet égard sans ambiguïté : Genesis ${ }^{27}$. Les dernières phrases de Salgado révèlent sa vision cyclique de l'existence, qui le remplit d'une impression d'être, par la nature, en contact avec l'éternité :

- En pensant que ces arbres, ils ont trois mois, ils vont atteindre leur climax à quatre cents ans, peut-être à partir d'ici on peut mesurer l'éternité, peut-être que l'éternité est mesurable. (1:22:32 - I:22:52)

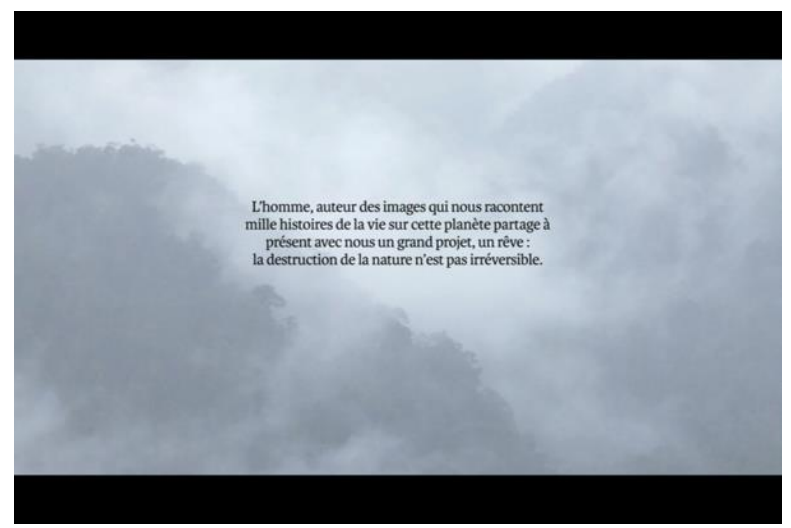

Fig. 30 : Photogramme du film Le sel de la Terre, Wim Wenders et Juliano Ribeiro Salgado, 2014 .

${ }^{26}$ Fondé en 1998, l'Instituto Terra est une organisation communautaire non gouvernementale à but non lucratif qui vise à restaurer l'écosystème de la région de la vallée du Rio Doce et, de manière élargie, à contribuer au développement des recherches sur l'environnement. Voir le site de l'organisme: http://www.institutoterra.org/pt br/ (consultation le 30 juin 2019).

${ }_{27}$ Genesis, Cologne, Taschen, 2013. 


\section{CONCLUSION}

En dépit de leurs traits communs, la similitude du dispositif technique et de l'entreprise heuristique (donner du sens par le cinéma à d'anciennes photographies), les trois œuvres confèrent une valeur et une signification bien différentes à ce qui peut apparaître comme un geste neutre, celui de restituer le contexte biographique et historique. Au sein même de chaque œuvre, la restitution occupe une place variable liée aux autres aspects de la temporalisation : dans $S i j$ 'avais quatre dromadaires, son importance augmente au cours du film, en reléguant le geste de création onirique de temporalités ou d'atemporalité à une première étape de maturation de la pensée; dans Ulysse, au contraire, après plusieurs tentatives, la restitution est abandonnée au profit d'une libre interprétation faisant la part belle à une vision mythographique; dans Le sel de la Terre, l'anecdotique n'est pas présenté comme un échec de l'interprétation de l'image photographique, néanmoins il est également dépassé pour laisser place à un diagnostic posé sur le réel représenté, qui correspond à une sortie de l'interprétation de l'œuvre photographique en tant qu'œuvre artistique.

La place de la restitution du contexte dans chaque œuvre est également liée à la conception que chacune offre du rapport entre pratique artistique et restitution qualitative du moment vécu. Après avoir restitué autant qu'elle le pouvait le contexte bio-historiographique de sa photographie, Varda en vient à abandonner le projet d'en restituer le moment dans sa singularité : il ne s'agit plus pour elle de donner à revivre l'instant grâce à l'anamnèse, mais de revenir à l'œuvre, à l'inépuisable richesse d'un symbole infiniment interprétable. En ce sens, son film est celui d'un échec assumé de la restitution du temps. En un autre sens, plus profond ou pour le moins conçu comme tel, Varda restitue le temps au-delà de l'anecdotique, en invoquant un temps transhistorique, qui rend justice au présent et à la possibilité d'envisager l'œuvre d'art sans son contexte bio-historiographique.

Le sel de la Terre est au contraire une célébration des pouvoirs quasi magiques de la photographie associée au cinéma pour ce qui est de restituer le temps des expériences vécues. Certaines séquences sont à la limite du supportable tant le dispositif fonctionne bien à cet égard. Sans aller jusqu'à la digression pensive, comme dans Si j'avais quatre dromadaires, Le sel de la Terre accorde de l'importance à l'anecdotique pour la lecture des ouvres photographiques. Cependant, si les tendances contraires alternent - appréciation de la singularité et vision d'ensemble, historicisme et vision anhistorique - , ce sont les deux secondes qui prennent le dessus. À la différence d'Ulysse, la favorisation de l'anhistoricité n'y est pas développée au profit de l'ouverture interprétative de l'œuvre photographique. C'est 
l'œuvre photographique qui, après avoir témoigné de l'humanité dans sa diversité, valorise la Terre comme Éden à restaurer. Dans son dernier temps, ce film sur la vie d'un photographe va jusqu'à sortir de la photographie, pour promouvoir un projet écologique consistant à restituer non pas de simples informations sur l'autrefois mais l'autrefois lui-même, purifié de l'histoire humaine. En cela, bien différemment d'Ulysse, il renouvelle aussi le sens de la restitution du temps ${ }^{28}$.

Le film de Chris Marker se situerait entre ces deux autres : quittant la contemplation des pouvoirs fantasmagoriques de l'œuvre d'art sans aller jusqu'à poursuivre l'œuvre hors de l'art, comme dans le cas du Sel de la Terre ${ }^{29}$. Sa penséeimages débute par la tentation de succomber aux charmes de la photographie comme technique de dé-temporalisation (ou de création de temporalités fictives), mais elle se comprend finalement au contraire comme organisation temporelle d'une multiplicité (d'images) pour envisager le monde, paradoxalement depuis des photographies, dans son inarrêtable temporalité.

${ }^{28}$ Il retrouve le sens archéologique et épigraphique du mot restituer : « rétablir dans son état premier, original, ce qui a subi des altérations » (nous citons à nouveau la définition donnée par le CNRTL).

29 Dans sa conférence «L'art n'a pas le privilège absolu », prononcée à l'Université de Liège le 9 mai 2oII, Georges Didi-Huberman marque la distance qui sépare le travail de Marker et Resnais dans Les statues meurent aussi, qu'il qualifie d'atlas parce qu'y est opérée une sortie hors du domaine de l'art, d'une démarche telle que celle de Malraux s'y cantonnant et constituant des albums. Mais c'est une chose de documenter (artistiquement) autre chose que l'art, et c'en est une autre de continuer l'œuvre en dehors ou au-delà de l'art, de lier un médium artistique à un médium non artistique. 


\title{
Temporaliser la photographie : Marker, Varda, Wenders- Salgado
}

\author{
Antoine Constantin Callle, Chercheur indéPEndant
}

\section{RÉSUMÉ}

À travers trois films qui reposent sur des photographies - Si j'avais quatre dromadaires de Chris Marker (1966), Ulysse d'Agnès Varda (1982) et Le sel de la Terre de Wim Wenders et Juliano Ribeiro Salgado (2014) - , cette contribution interroge la valeur et le sens de la restitution du temps. Nous considérons la part que joue dans le film la restitution du contexte bio-historiographique de la photographie, mais aussi la capacité de l'œuvre photocinématographique à rendre sensible le temps. De quel temps s'agit-il alors ? Celui du réel photographié ou celui inventé par l'œuvre ? Peut-on restituer ce temps réel et singulier de l'instant photographié, et est-ce cette restitution qui donnera tout son sens à la photographie ? Quelle importance faut-il donner à la singularité du moment ? L'enjeu de la restitution du temps ne dépasse-t-il pas celui de la restitution de cette singularité ?

\section{ABSTRACT}

Through a discussion of three films based on photographs-Chris Marker's If I Had Four Camels (1966), Agnès Varda's Ulysses (1982), and Wim Wenders and Juliano Ribeiro Salgado's The Salt of the Earth (2014)-this essay questions the value and meaning of rendering time. It considers the importance of the photograph's biohistoriographic context as well as the ability of the photo-cinematographic work to render time perceptible. But which time is thus concerned? The one of the photographed reality or the one invented by the work of art? Is it possible to render the real and singular time of the photographed instant, and is it this rendering that bestows meaning to the photograph? What importance should be given to the singularity of the moment? Does the challenge of rendering time transcend that of rendering singularity?

\section{NOTE BIOGRAPHIQUE}

Antoine Constantin Caille a obtenu un doctorat en études francophones à l'Université de Louisiane en 20I5, puis a enseigné dans les départements de langues modernes de l'Institut de technologie de Géorgie et du Collège de William \& Mary en Virginie. Il est actuellement professeur de philosophie et de lettres modernes à l'École Al Jabr de Casablanca. Ses articles parus dans différentes revues universitaires portent sur la théorie critique, la littérature de langue française, la traductologie, le cinéma et les arts visuels. On 
peut accéder à sa page personnelle à partir du lien ci-dessous : https://independent.academia.edu/AntoineConstantinCaille. 